Supplementary Information

\title{
UNEXPECTED REDUCTION OF INDOLE DOUBLE BOND IN MITRAGYNINE USING $N$-BUTYLSILANE AND CATALYTIC TRIS(PENTAFLOROPHENYL)BORANE
}

Goh Teik Beng*, Ramu Meesala, Mohd Nizam Mordi and Sharif Mahsufi Mansor.

Centre of Drug Research, Universiti Sains Malaysia, Minden 11800, Penang, Malaysia.

E-mail : gohteikbeng@yahoo.com Phone : 6046533273 Fax : 6046568669 


\section{Equipments}

Various analytical methods such as UV-Visible, IR, NMR, mass spectroscopy, CHN analysis, HPTLC and DSC melting point were carried out to characterize the isolated pure (E)-methyl 2-((2S,3S,12bS)-3-ethyl-8-methoxy-1,2,3,4,6,7,7a,12,12a,12b decahydroindolo[2,3a]quinolizin-2-yl)-3-methoxyacrylate free base. UV-Visible spectroscopy was carried out using Shimadzu UV 160-A double beam spectrophotometer (Kyoto, Japan) using the HPLC grade methanol as a blank. FTIR spectra were recorded using $\mathrm{KBr}$ pellets by a Thermal Scientific Nicolet 6700 spectrophotometer with Omnic software (Franklin, USA). ${ }^{1}$ H-NMR and ${ }^{13} \mathrm{C}-\mathrm{NMR}$ were performed using $400 \mathrm{MHz}$ and $100 \mathrm{MHz}$ Bruker spectrometer (Karlsruhe, Germany), respectively. One-dimensional and two-dimensional NMR experiments were carried out on a Bruker 600 FT-NMR (Darmstadt, Germany) operating at $25^{\circ} \mathrm{C}$. The samples were dissolved in $\mathrm{CDCl}_{3}$ and the chemical shifts were recorded with reference to that of tetramethylsilane (0, ppm). GC-MS spectra were taken using HP 6890A GC system (Santa Clara, USA) with HP-5MS Polyimide coated capillary column (30 m x $0.25 \mathrm{~mm}$ i.d. $\mathrm{x} 0.1 \mu \mathrm{m})$, heated from $100^{\circ} \mathrm{C}$ to $280^{\circ} \mathrm{C}$ at $10^{\circ} \mathrm{C} / \mathrm{min}, 10 \mathrm{Kpa}$ helium at 1.00 $\mathrm{mL} / \mathrm{min}$ of flow rate and an injection volume of $\mathrm{I} \mu \mathrm{L}$ with split ratio of $5: 1$. CHN elemental analysis was conducted using Perkin Elmer CHN Analyzer Model 2400-2 (Massachusetts, USA). HPTLC study was performed on a $0.50 \mathrm{~mm}$ thick Silica gel $60 \mathrm{G}_{254}(10 \mathrm{~cm}$ x $20 \mathrm{~cm})$ using a mobile phase hexane:ethyl acetate $(92: 8 \mathrm{v} / \mathrm{v})$. TLC plates were then visualized under UV light (254 and $366 \mathrm{~nm})$. CD spectra was measured at 250C on a Jasco-715 CD spectrapolarimeter. All CD spectra were recorded with the standard sensitivity, a band width of $2.0 \mathrm{~nm}$, a data pitch of $0.5 \mathrm{~nm}$ and a scan velocity of $20 \mathrm{~nm} / \mathrm{min}$. The CD spectra was calibrated using (1R)-(-)-10-camphorsulphonic acid and (R)-(-)-Pantolactones according to Miles et al., 2008. The solvent was always recorded on the same day as the analytical sample. Evaluation of the data was performed with the program "Spectra Analysis" of the Jasco-715 
instruments. The wavelength Range was from $190 \mathrm{~nm}$ to $350 \mathrm{~nm}$. The scan Speed is Medium and response time was Medium. The number of scans was 16-32. DSC Pyris 6 (Mettler Toledo, Switzerland) differential scanning calorimetry with STARe software (Mettler Toledo) was used for melting point measurement. Indium (melting point $156.6^{\circ} \mathrm{C}$ and melting enthalpy $28.45 \mathrm{~J} / \mathrm{g}$ ) was chosen for standard calibration of instrumental cell constant and temperature, in order to obtain correct and precise determination result. The DSC thermogram was plotted from $30{ }^{\circ} \mathrm{C}$ to $300{ }^{\circ} \mathrm{C}$ and the analysis was performed at a heating rate of $10{ }^{\circ} \mathrm{C}$ per minute. The glass transition temperature, Tg was measured using the mid point method. Approximately $4.500 \pm 0.500 \mathrm{mg}$ of samples was weighed precisely and transferred accurately into the DSC crucible pans with seal with pre-pin lid.The crucibles was Capped and packed by moulding machine before fixing into the DSC furnace. After cooling by liquid nitrogen $(99.99 \%)$, the sample was heated from $30 \circ \mathrm{C}$ to $300{ }^{\circ} \mathrm{C}$ at the velocity of $10^{\circ} \mathrm{C} / \mathrm{min}$. The heating and cooling stages were running at $20 \mathrm{~mL} / \mathrm{min}$ purge gas and $50 \mathrm{~mL} / \mathrm{min}$ shield gas (nitrogen gas) ambience. The method of $30{ }^{\circ} \mathrm{C}$ to $300{ }^{\circ} \mathrm{C} @ 10{ }^{\circ} \mathrm{C} / \mathrm{min}$ N2 with Blank was used. A background calibration with blank empty pan at both reference and sample sensors was performed prior to the analysis. 
A) Silane Reduced Mitragynine

1. ${ }^{1} \mathrm{H}$ NMR-Aliphatic Expanded Region
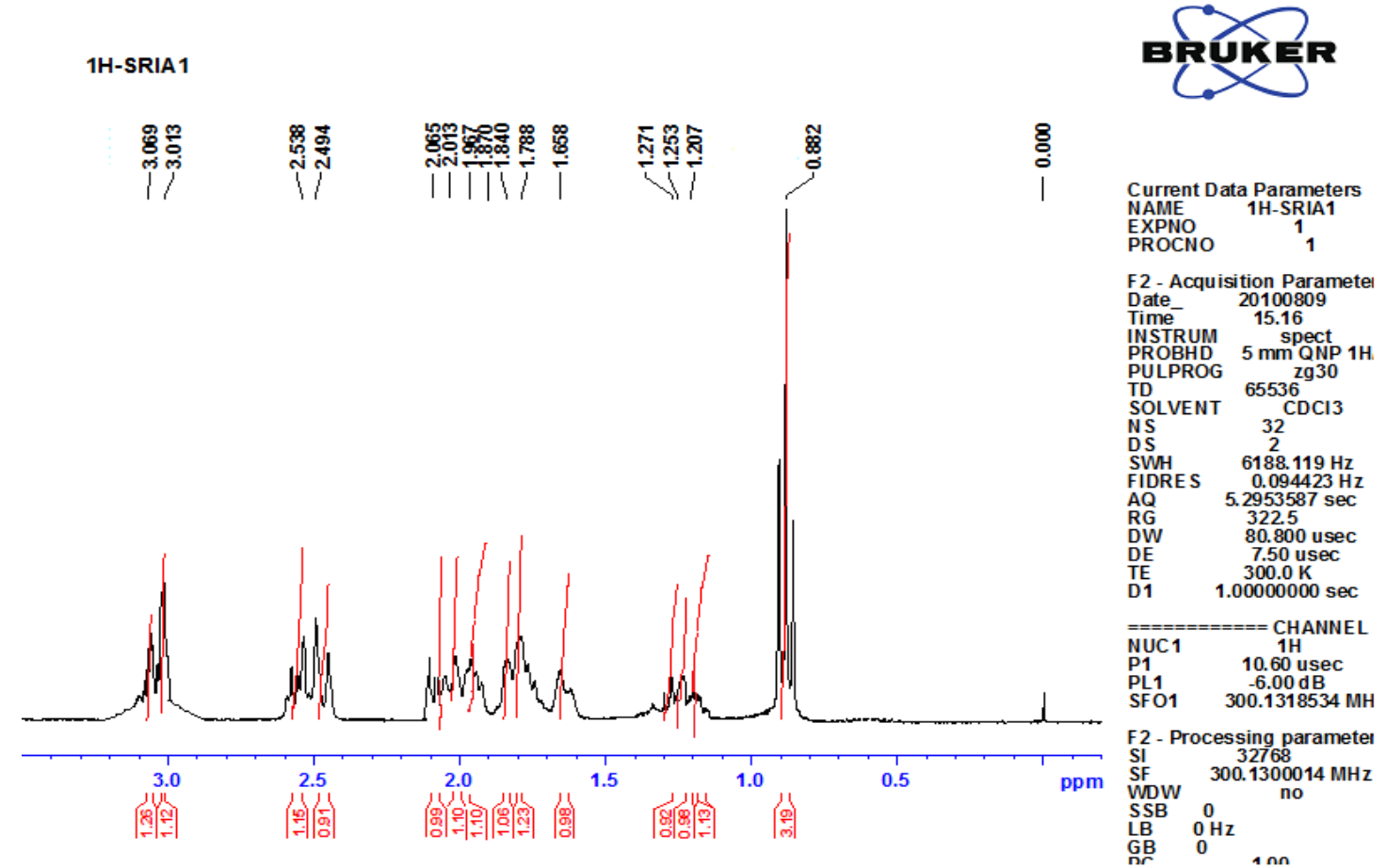

2. ${ }^{1} \mathrm{H}$ NMR - Expanded Aromatic Region

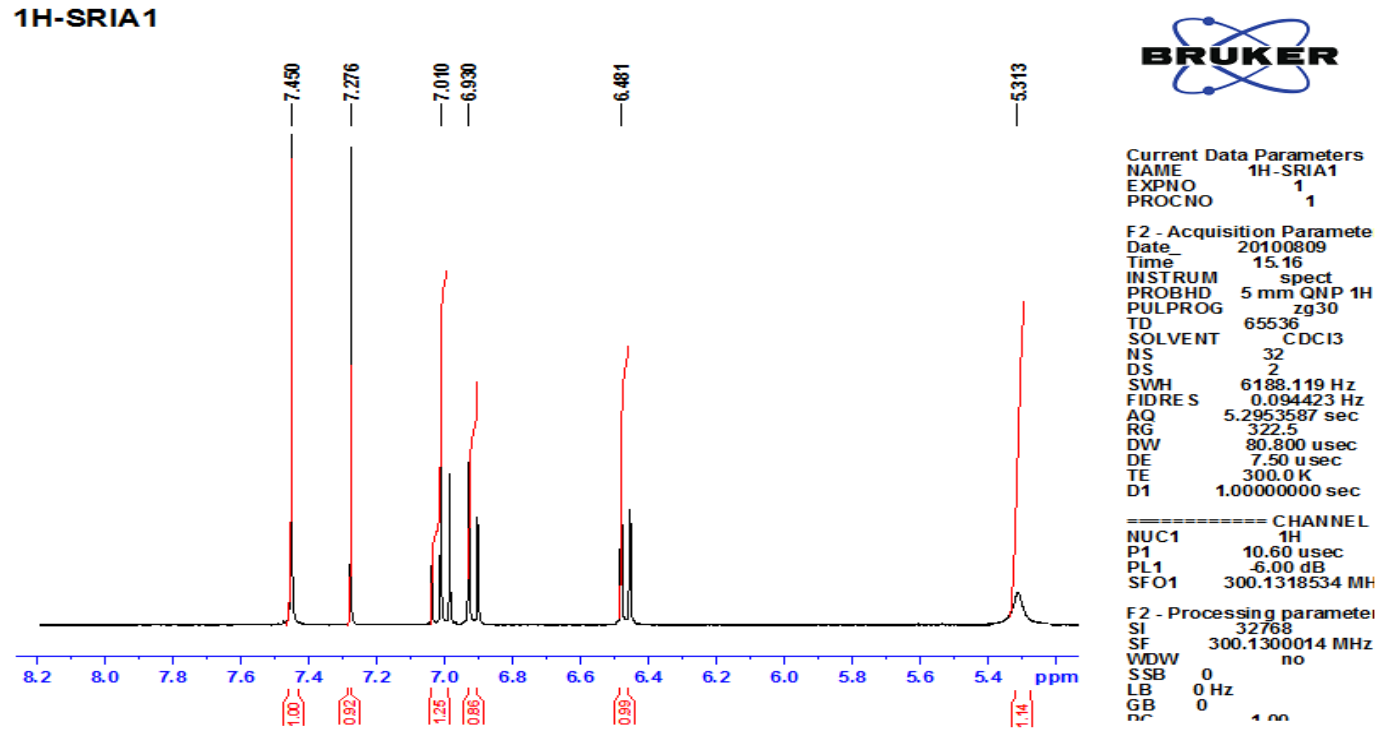


3. ${ }^{1} \mathrm{H}$ NMR -Methoxy group

1H-SRIA1
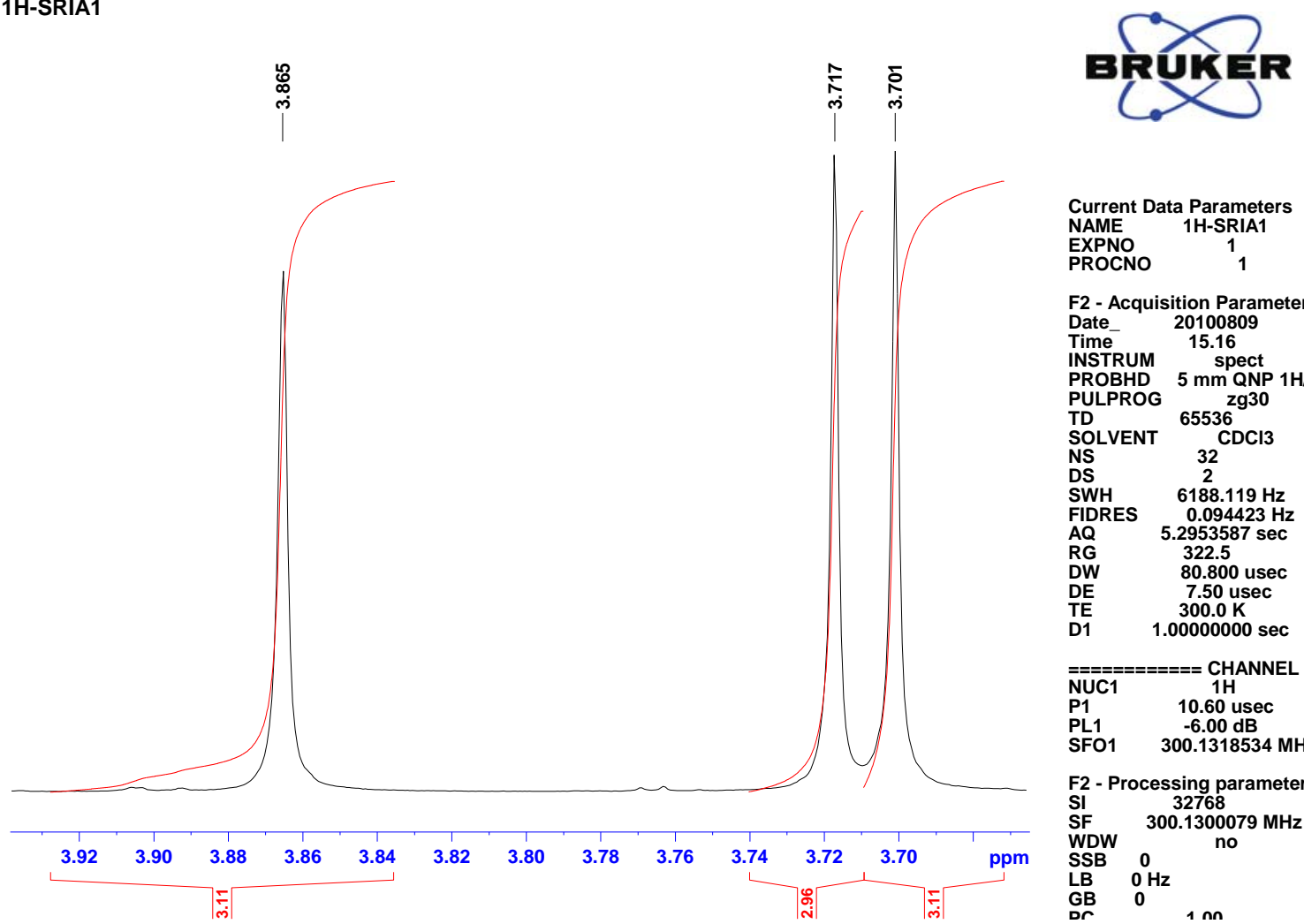

Current Data Parameters

NAME 1H-SRIAI

$\begin{array}{ll}\text { EXPNO } & 1\end{array}$

1

F2 - Acquisition Paramete

Date__ 20100809

$\begin{array}{ll}\text { Time } & 15.16 \\ \text { INSTRUM } & \text { spect }\end{array}$

spect
PROBHD $5 \mathrm{~mm}$ QNP 1H

$5 \mathrm{~mm}$ QNP
PULPROG

TD $\quad 65536$

NS

DS

${ }_{32} \mathrm{CDCl} 3$

$\quad 22.119 \mathrm{~Hz}$

$\begin{array}{lr}\text { FIDRES } & 0.094423 \mathrm{~Hz} \\ \text { AQ } & 5.2953587 \mathrm{sec}\end{array}$

RG $\quad 322.5$

$\quad \mathbf{8 0 . 8 0 0}$ usec

DE $\quad 7.50$ usec

D1 $1.00000000 \mathrm{sec}$

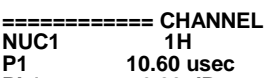

P1 10.60 usec

SFO1 $\quad 300.1318534 \mathrm{MH}$

F2 - Processing paramete

SI $\quad 32768$

SF $\quad 300.1300079 \mathrm{MHz}$

WDW no

SSB 0

$\begin{array}{ll}\mathrm{LB} & 0 \mathrm{~Hz} \\ \mathrm{~GB} & 0\end{array}$

4. ${ }^{1} \mathrm{H}$ NMR full

1H-SRA2 in $\mathrm{CDCl} 3$ Proton $\mathrm{CDCl} 3$

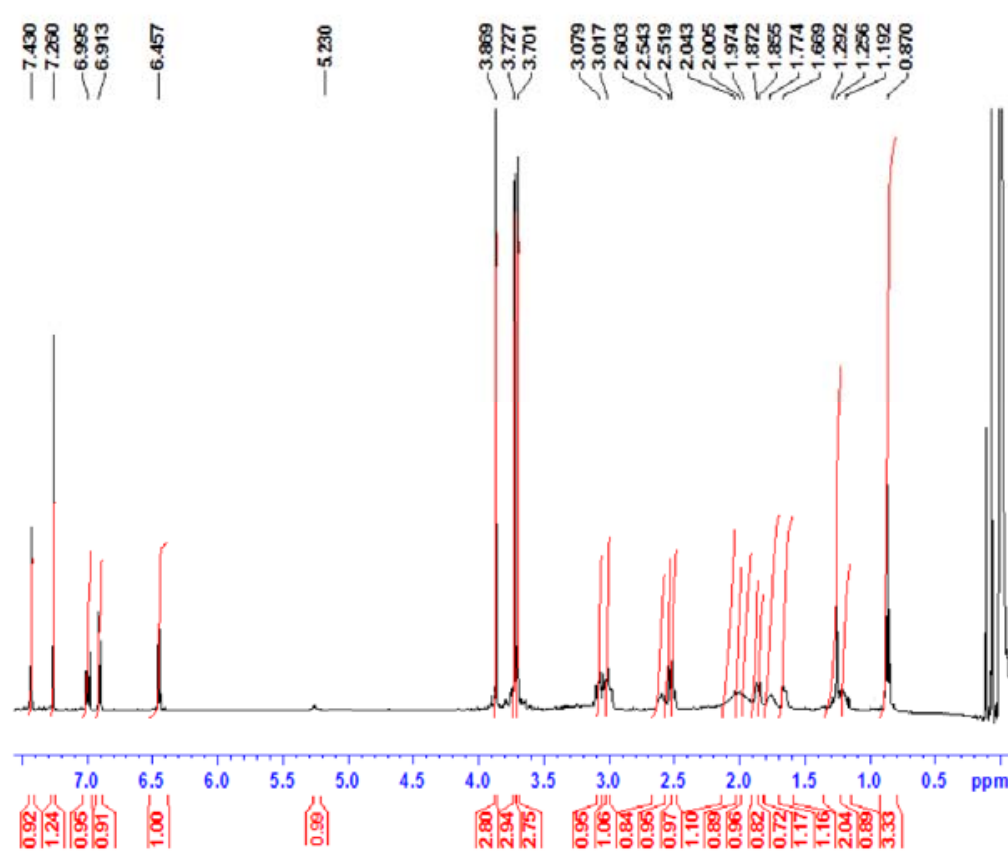

हरUKER
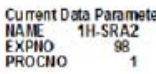

F2. Acquisition Paraneler

Date- 2010082

PROBHO $5 \mathrm{~mm}$ PABBO BS

PULPROG $65536^{2930}$

SOLVENT CDC

$\begin{array}{ll}115 & 16 \\ \text { DS } & 2 \\ \text { SWH } & 10330.578 \mathrm{~Hz} \\ & \end{array}$

$\begin{array}{ll}\text { FIDRES } & 0.15763 \mathrm{BH} \\ \text { AO } & 3.1719923 \mathrm{sec}\end{array}$

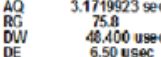

$\begin{array}{ll}\text { DE } & 6.50 \mathrm{Usec} \\ \text { TE } & 24.5 \mathrm{~K} \\ \mathrm{D} & 10000000\end{array}$

-...-- CHANMEL 11 -.....

${ }_{\mathrm{P1}}^{\mathrm{MUC1}} \quad 14 \mathrm{H}$

$\begin{array}{ll}\text { PLWH } & 15.00000000 \mathrm{~W} \\ \text { SFO1 } & 500.1330685 \mathrm{MHz}\end{array}$

F2. Processing parameters

SI $\quad 500.1300144 \mathrm{MHz}$

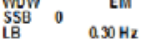


$5{ }^{13} \mathrm{C}$ NMR Full Spectrum

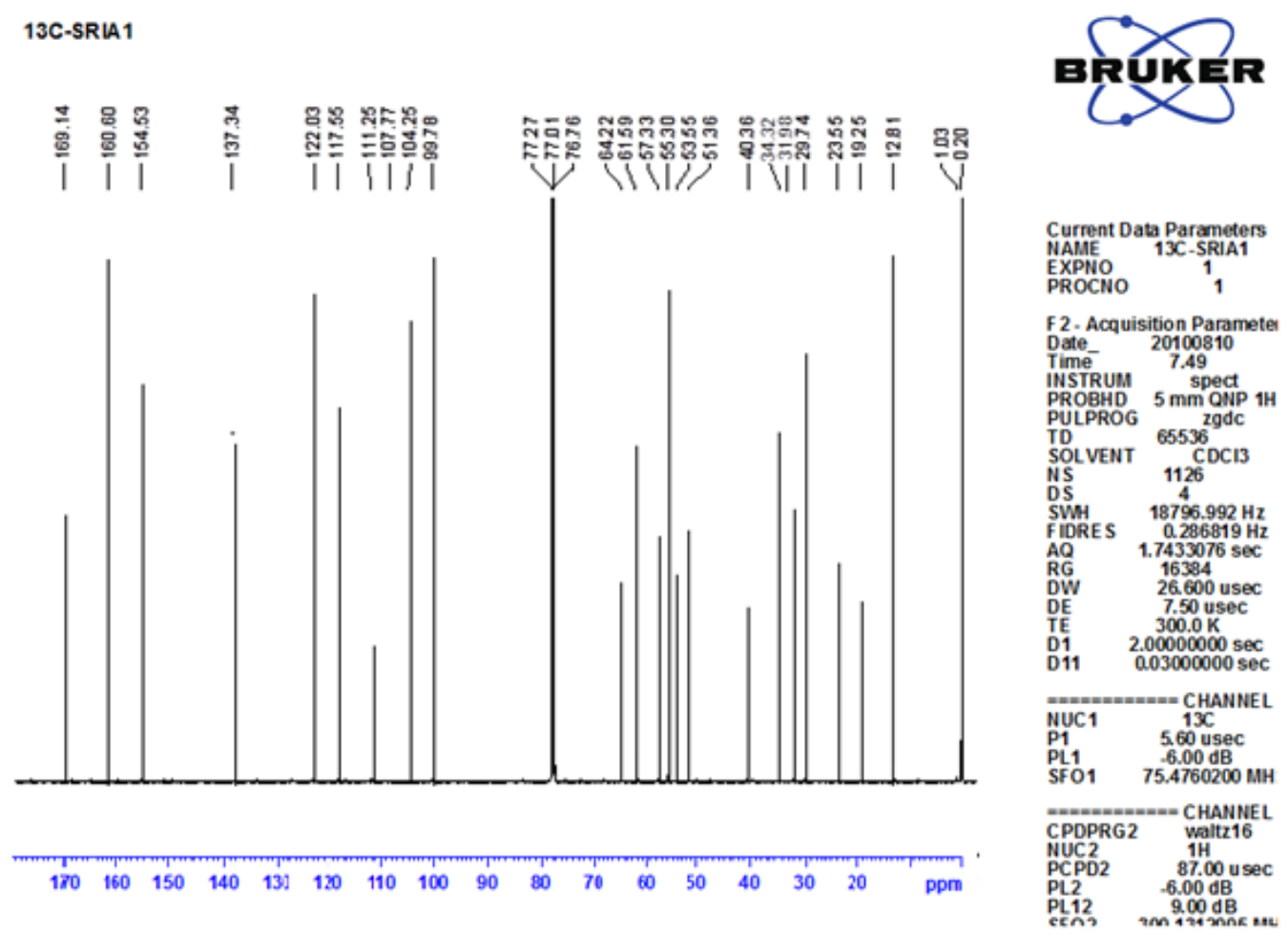

6. Dept-90 
DEPT90 SR1A in CDCI3

C13DEPT90
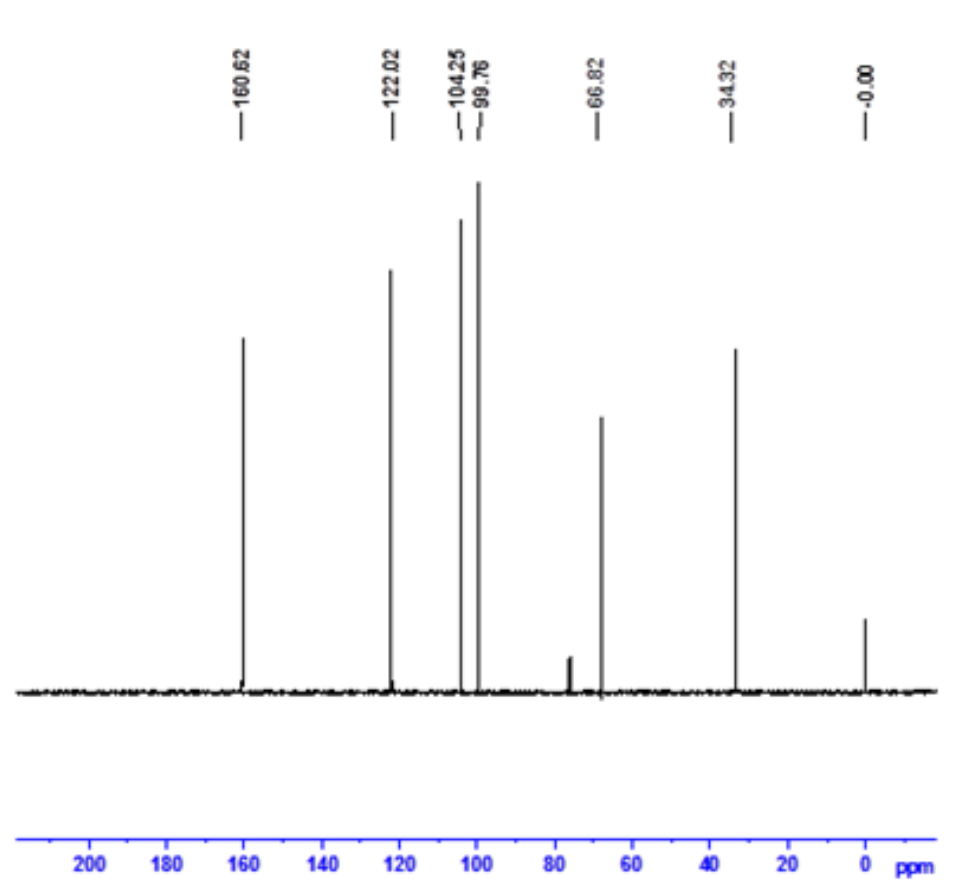

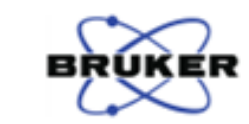

Current Data P arameters

NAME SR1ADEPT90

$\begin{array}{ll}\text { EXPNO } & 16 \\ \text { PROCNO } & 1\end{array}$

F2 - Acquisition P arameters

Date_ 20100812

INSTRUM spect

PROBHD $5 \mathrm{~mm}$ PABBO BI

PULPROG DEPT90

TD 65536

SOLVENT CDCI3

NS 256

SWH $\quad 29761.904 \mathrm{~Hz}$

$\begin{array}{lr}29761.904 \mathrm{~Hz} \\ \text { FIDRE S } & 0.454131 \mathrm{~Hz}\end{array}$

$\mathrm{AQ} \quad 1.1010548 \mathrm{sec}$

RG $\quad 188.56$

DW $\quad 16.800$ usec

DE 6.50 usec

TE $296.8 \mathrm{~K}$

CNST2 145.0000000

CNST11 $\quad 1.0000000$

D1 $2.00000000 \mathrm{sec}$

D20 $0.00689655 \mathrm{sec}$

$==x==x$ CHANNEL $f 1==$

NUC1 $13 \mathrm{C}$

P1 10.25 usec

P2 20.50 usec

PLW1 74.00000000 W

SFO1 $125.7703643 \mathrm{MH}$

$=======\mathrm{CHANNEL} 2==$

CPDPRG2 waltz16

NUC2 $21 \mathrm{H}$

\section{Dept- 45}

DEPT45 SR1A in CDCl3

C13DEPT45 CDCl3
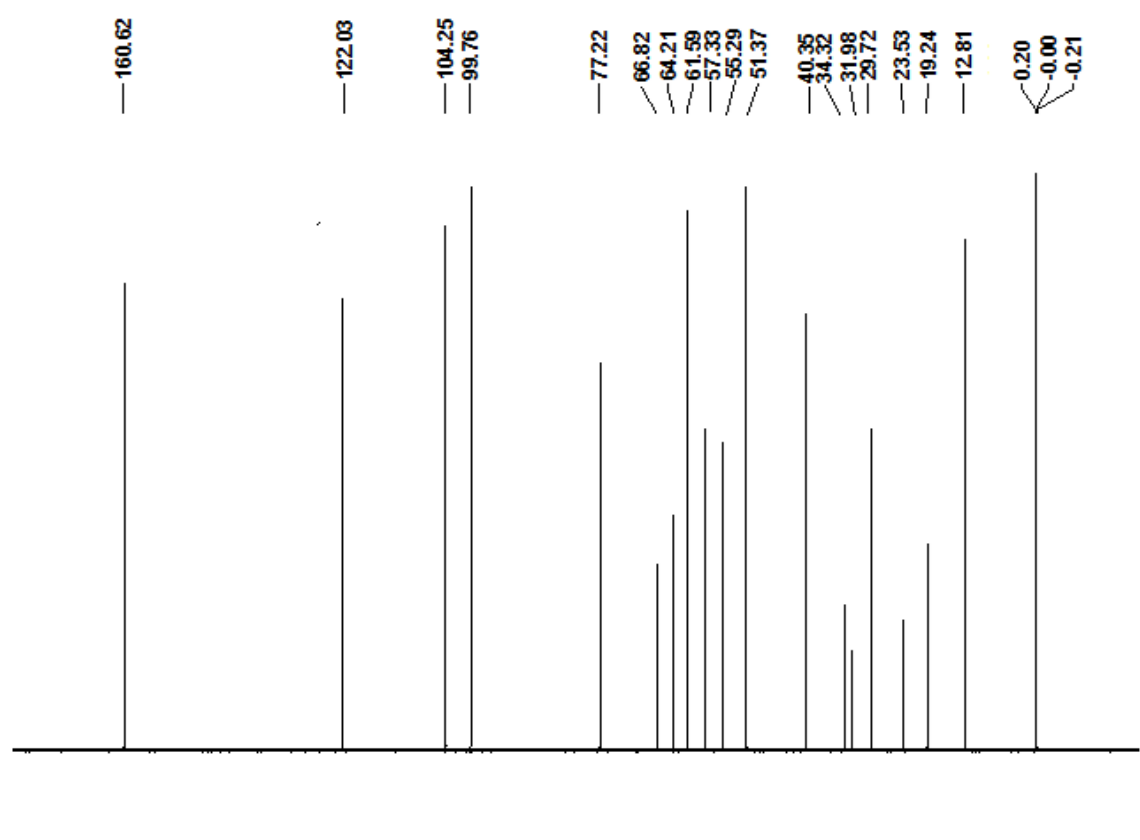

$\begin{array}{lllllllll}170 & 160 & 150 & 140 & 130 & 120 & 110 & 100 & 90\end{array}$

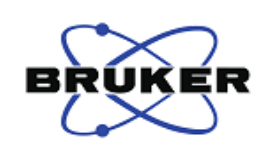

Current Data Parameters NAME SR 1ADEPT 45 PROCNO 19

F2 - Acquisition Parameters

$\begin{array}{lc}\text { Date_ } & 20100812 \\ \text { Time } & 9.52\end{array}$ $\begin{array}{ll}\text { IN STRUM } & \text { spect } \\ \text { PROBHD } & 5 \mathrm{~mm} \text { PABBO BB }\end{array}$ PROBHD $5 \mathrm{~mm}$ PABBO BB
PULPROG
DEPT45

SOLVENT ${ }^{65536} \mathrm{CDC} 3$ $\begin{array}{lc}\text { SOLVENT } & \text { CDCl } \\ \text { NS } & 256 \\ \text { DS } & 4\end{array}$

$\begin{array}{lc}\text { DS } & 4 \\ \text { SWH } & 29761.904 \mathrm{~Hz}\end{array}$ FIDRES $\quad 0.454131 \mathrm{~Hz}$ AQ $\quad 1.1010548 \mathrm{sec}$ RG $\quad \mathbf{1 8 8 . 5 6}$ DW $\quad 16.800$ usec DE $\quad 6.50$ usec $\begin{array}{ll}\text { TE } & 296.8 \mathrm{~K} \\ \text { CNST2 } & \mathbf{1 4 5 . 0 0 0 0 0 0 0}\end{array}$

CN ST2 $\quad 145.0000000$

CN ST11 1.0000000 $\begin{array}{ll}\text { D1 } & 2.00000000 \mathrm{sec} \\ \text { D20 } & 0.00689655 \mathrm{sec}\end{array}$ $=======$ CHANNEL $\mathbf{f} 1===$ P1 10.25 usec $\begin{array}{ll}\text { P1 } & 10.25 \text { usec } \\ \text { P2 } & 20.50\end{array}$ $\begin{array}{ll}\text { P2 } & 20.50 \text { usec } \\ \text { PLW1 } & 74.00000000 \mathrm{~W}\end{array}$ $=====$ CHANNEL $f 2===$ CPDPRG 2 waltz $\begin{array}{ll}\text { NUC2 } & 1 \mathrm{H} \\ \text { PC.Pn? } & \end{array}$ 
8. Dept- 135

DEPT135 in CDCI3

C13DEPT135 $\mathrm{CDCl} 3$

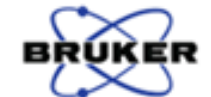

厂

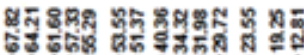

$\begin{array}{lc}\text { Current Data Parameters } \\ \text { NAME } & \text { SR1ADEPT135 } \\ \text { EXPNO } & 18 \\ \text { PROCNO } & 18\end{array}$

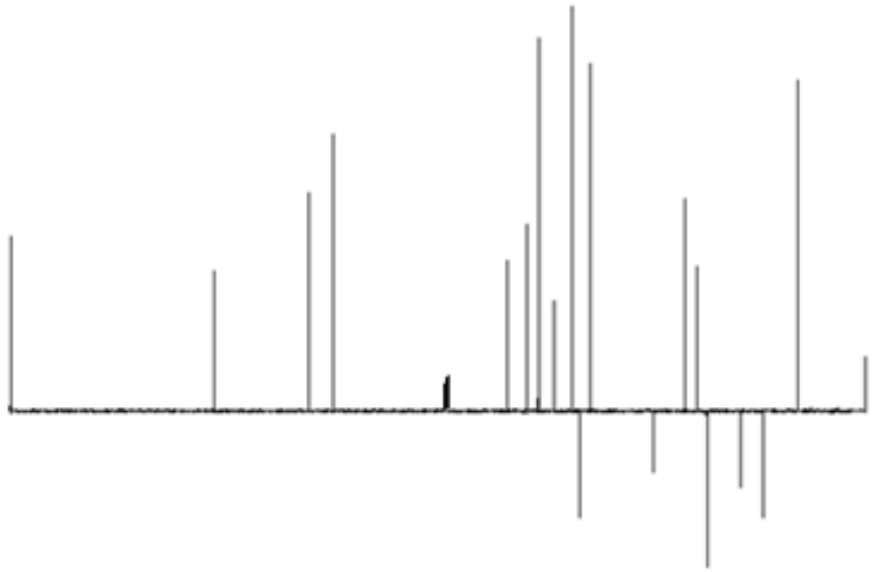

F2 - Aquisition Parameters

Date_ 20100812

Time -9.36

NSTRUM

PROBHD $5 \mathrm{~mm}$ PABBO BB.

PULPROG DEPT135

TD 65536

SOLVENT ${ }_{256} \mathrm{CDCl}$

DS 4

SWH $\quad 29761.904 \mathrm{~Hz}$

FIDRE S $\quad 0.454131 \mathrm{~Hz}$

$\mathrm{AQ} \quad 1.1010548 \mathrm{sec}$

RG $\quad 188.56$

DW $\quad 16.800$ usec

DE $\quad 6.50$ usec

TE $296.8 \mathrm{~K}$

CNST2 145.0000000

CNST11 1.0000000

D1 $2.00000000 \mathrm{sec}$

D20 $\quad 0.00689655 \mathrm{sec}$

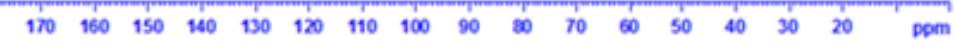

$==x==x=$ CHANNEL $f 1=x==$

NUC1 $13 \mathrm{C}$

$\begin{array}{ll}\text { P1 } & 10.25 \text { usec } \\ \text { P2 } & 20.50 \text { usec }\end{array}$

PLW1 74.00000000 W

SFO1 $125.7703643 \mathrm{MHz}$

\section{9. $A P T{ }^{13} \mathrm{C}$}

APT 13C SR1A in $\mathrm{CDCl}_{3}$ C13APT CDCl3
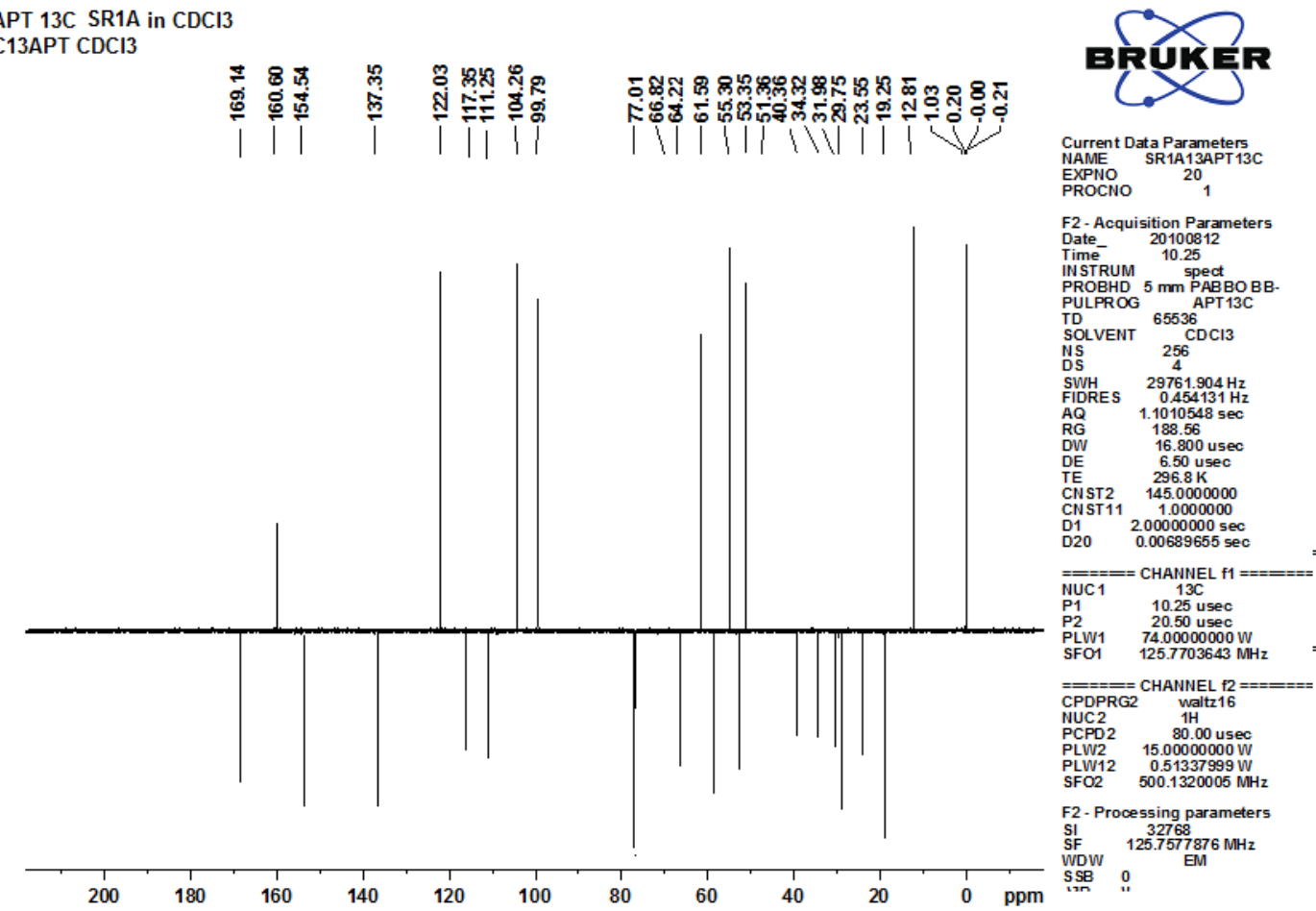
10. HSQC-Aromatic Expanded Region

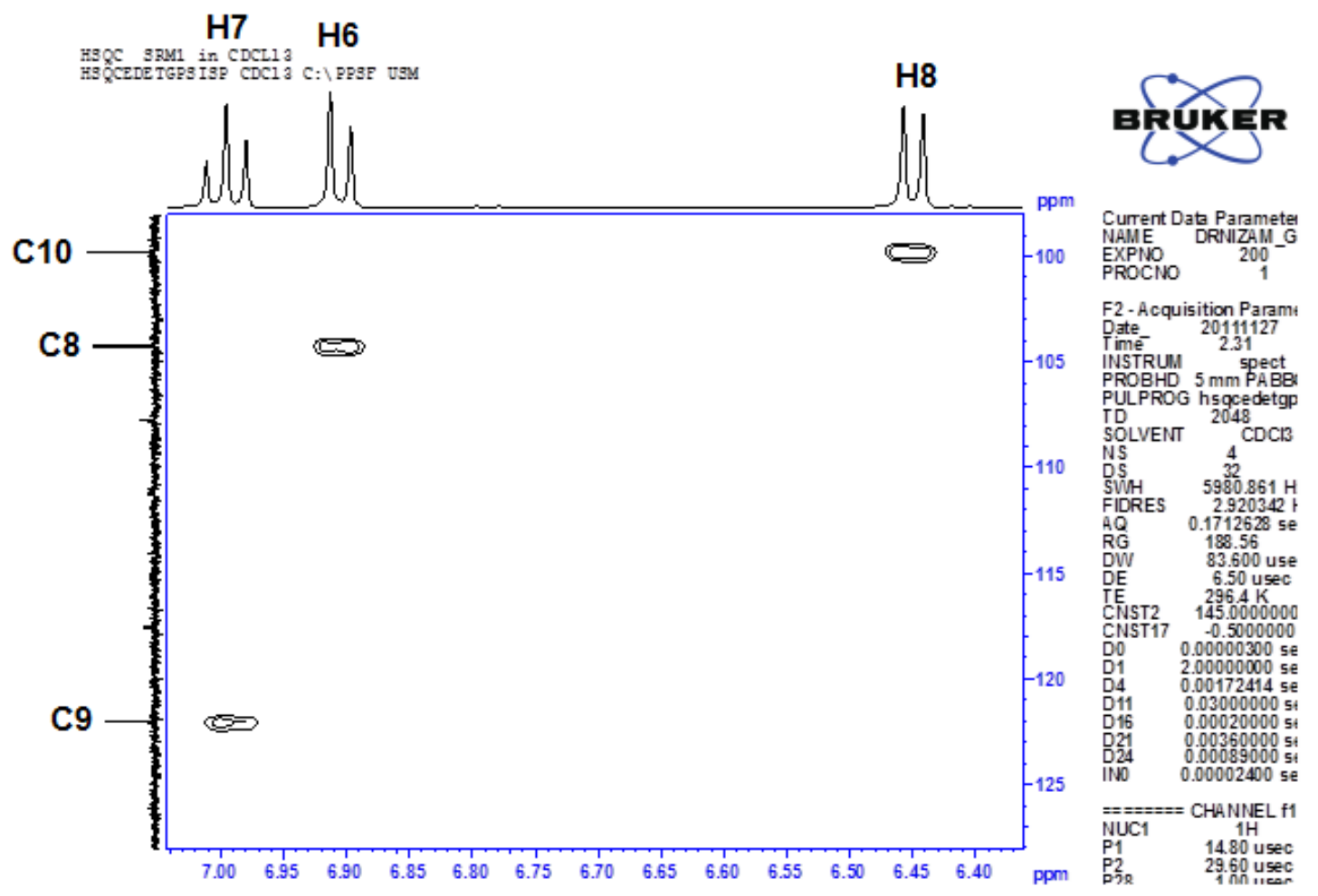

11. HSQC-Aromatic Expanded Region

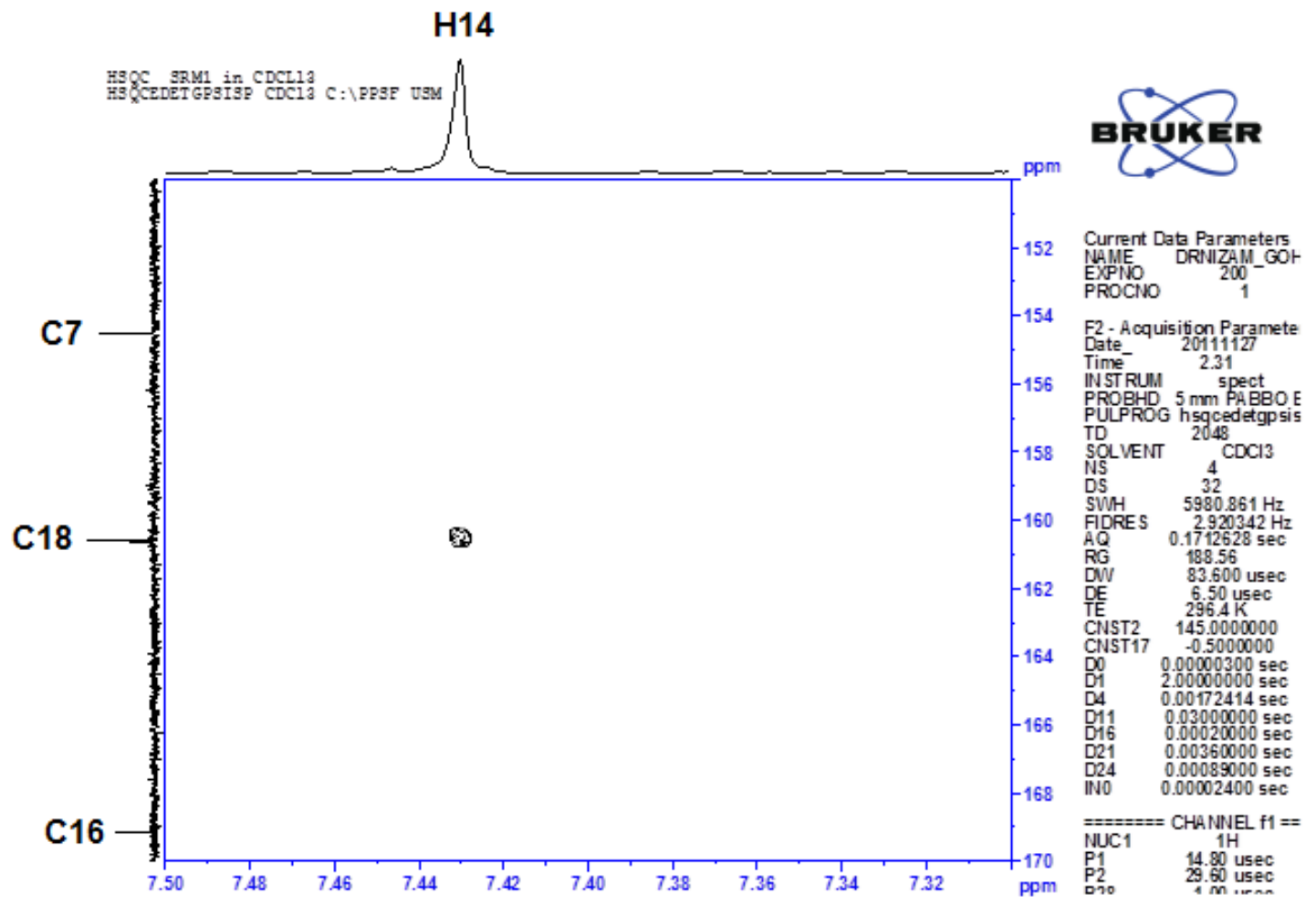




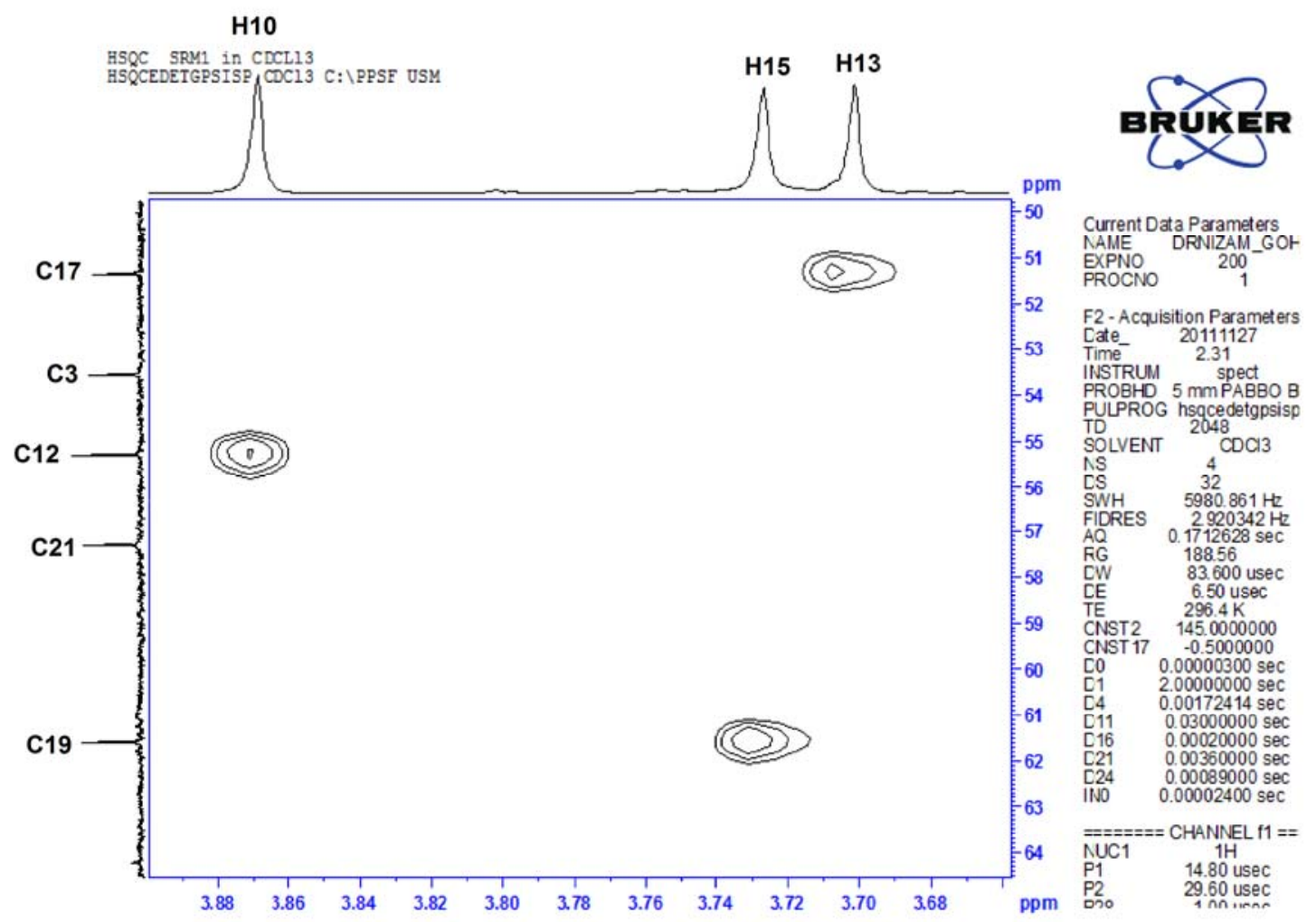

13. HSQC -Expanded aliphatic Region

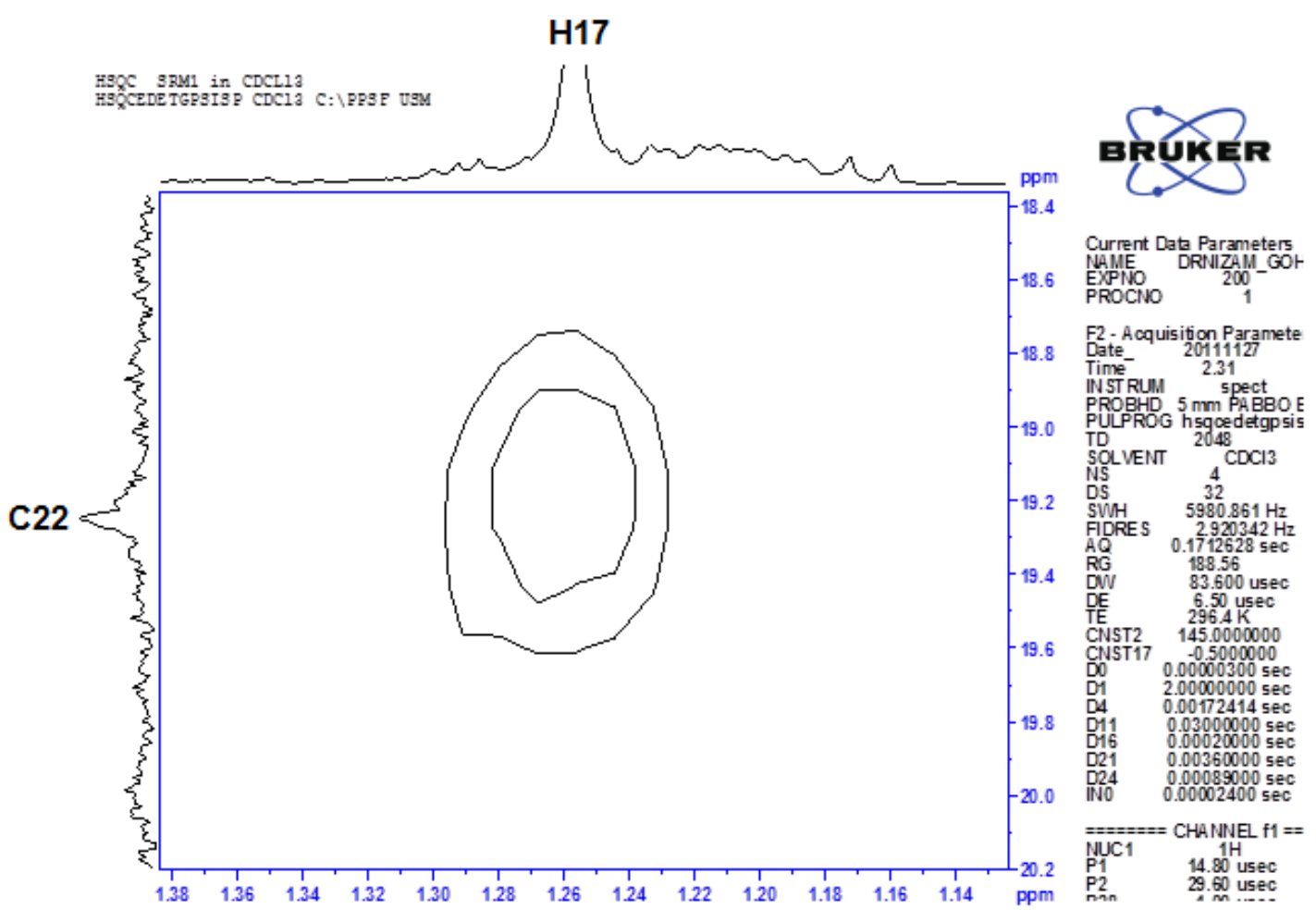


14. HSQC-Expanded Aliphatic Region

\section{H18}

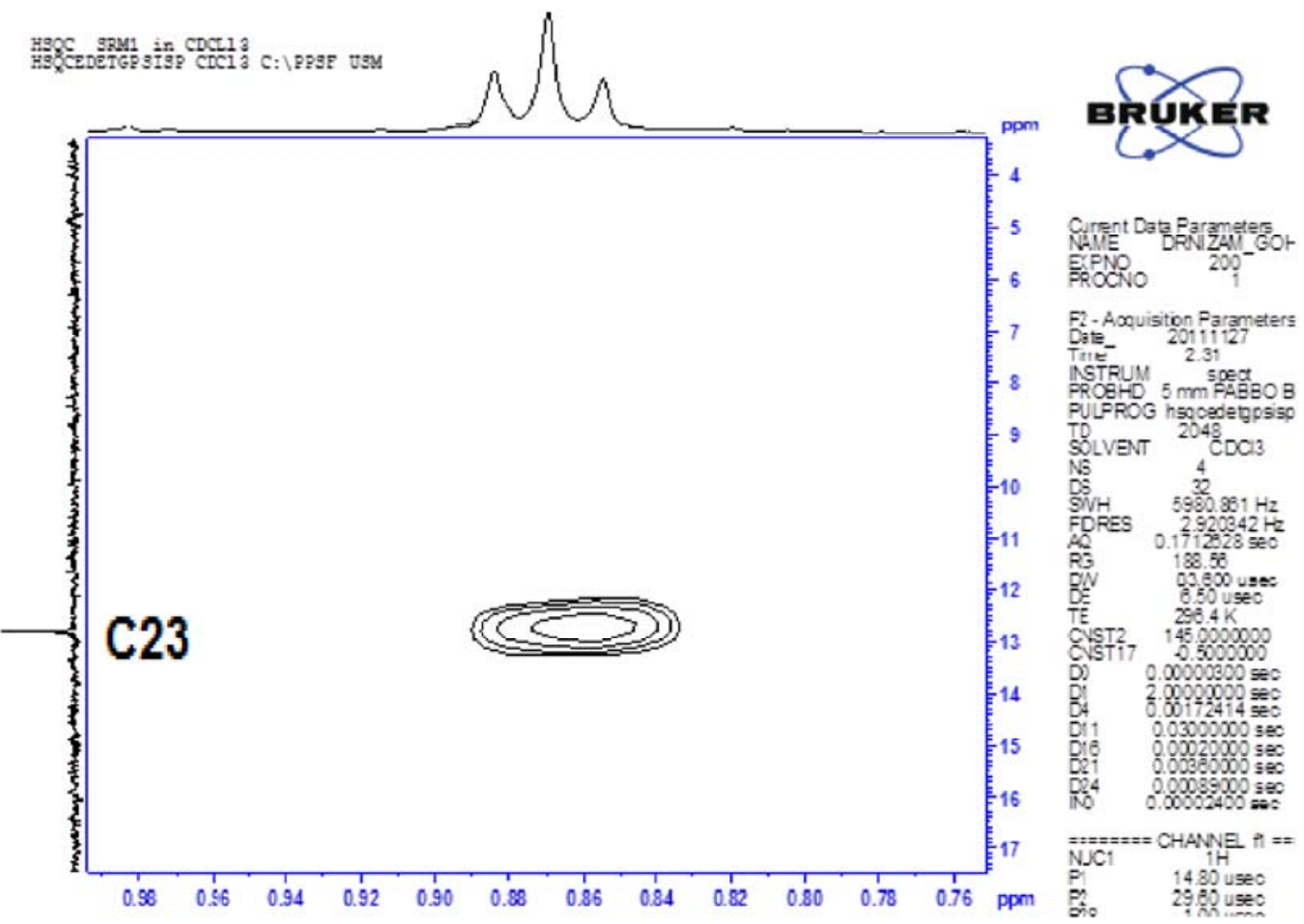

15 HSQC -Aliphatic Expanded Region

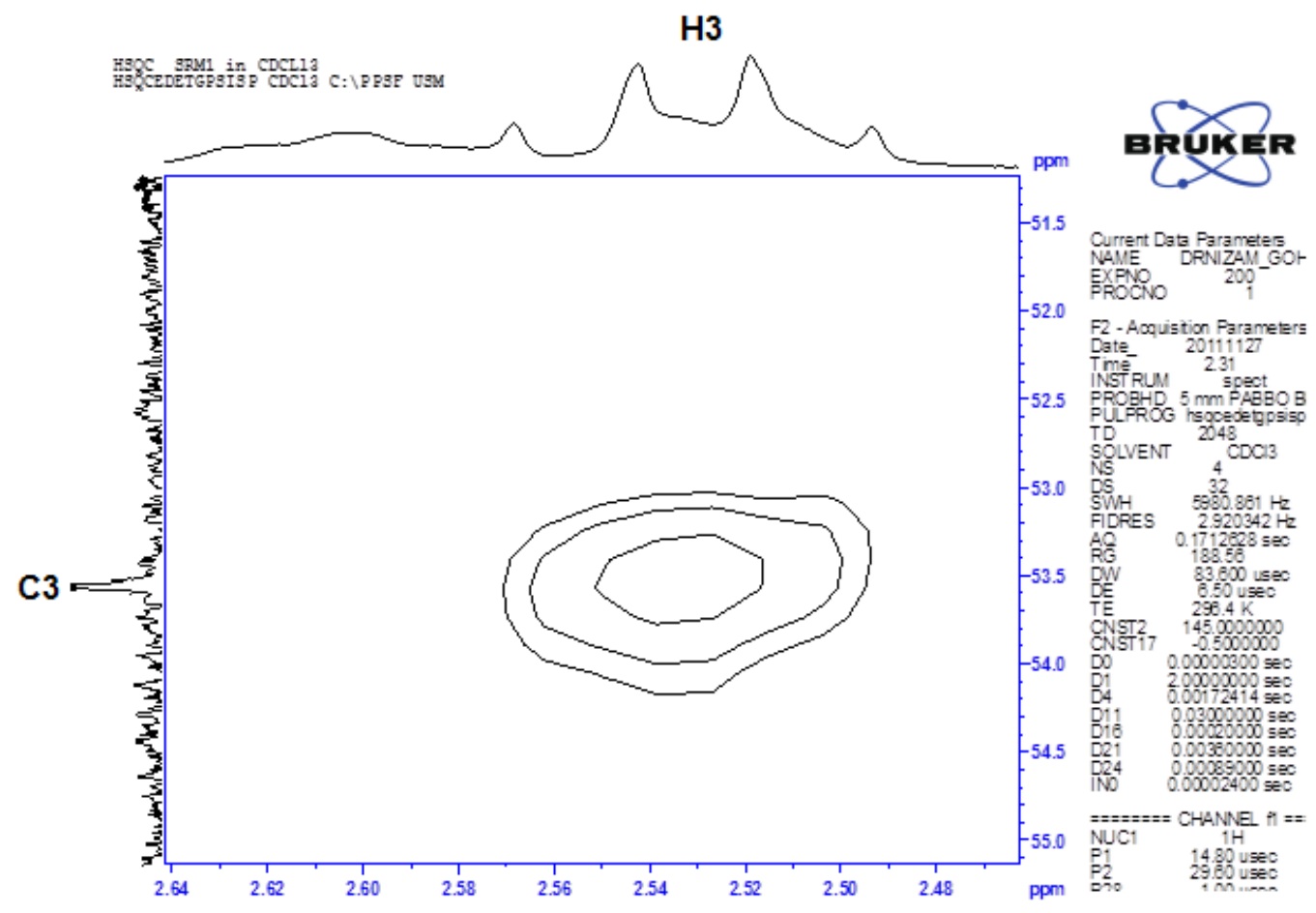


16. HSQC-Aliphatic Expanded Region

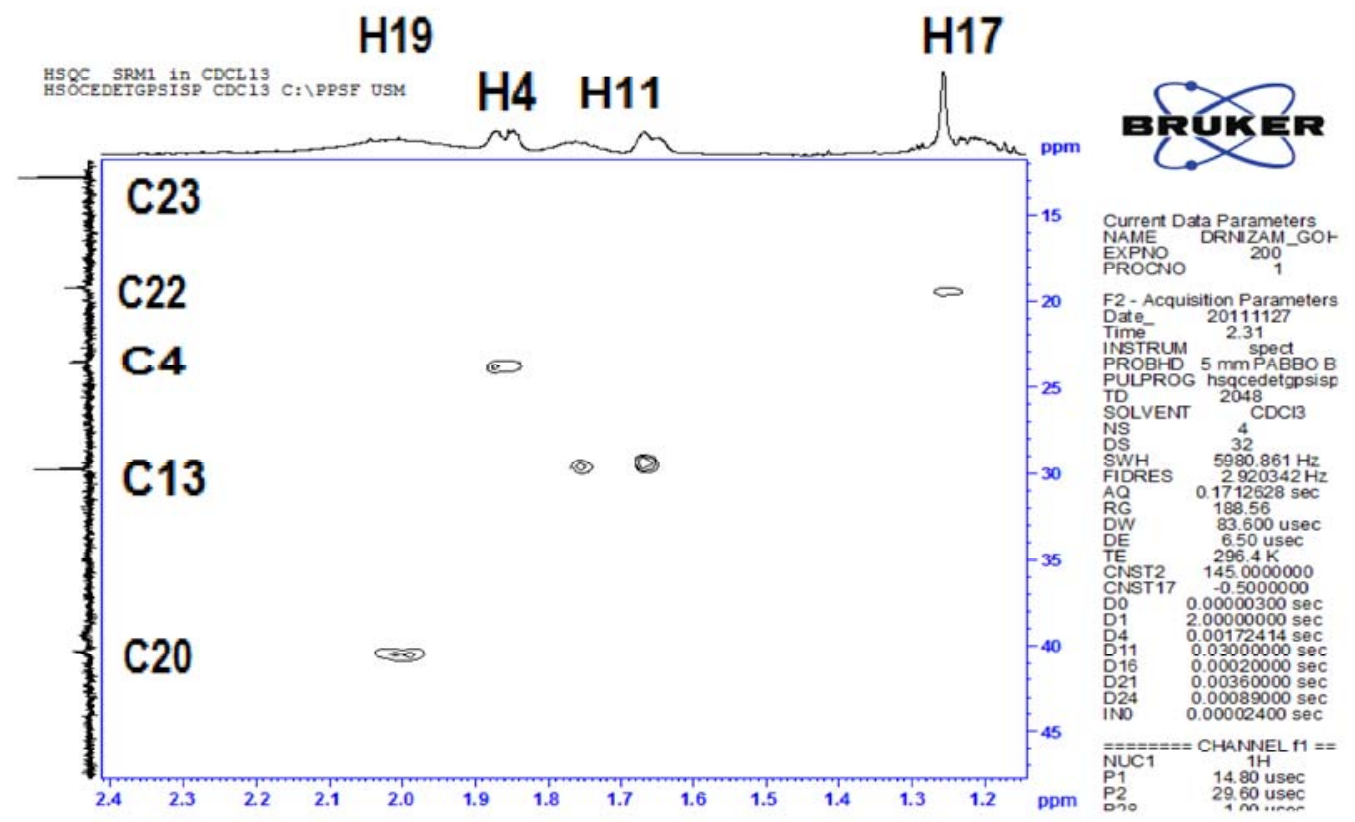

17. HSQC-Aliphatic Expanded Region

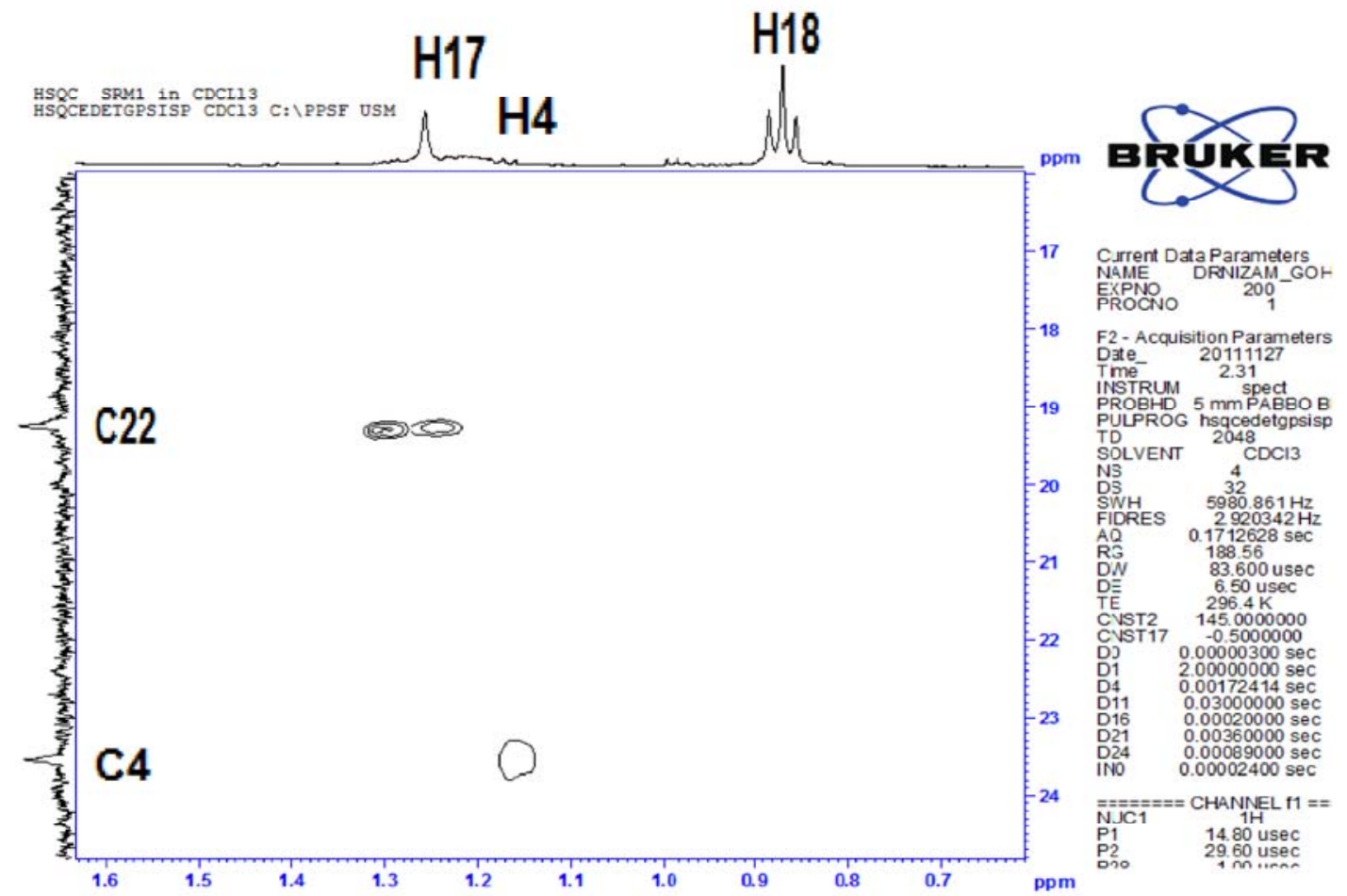


18. HSQC-Aliphatic Expanded Region

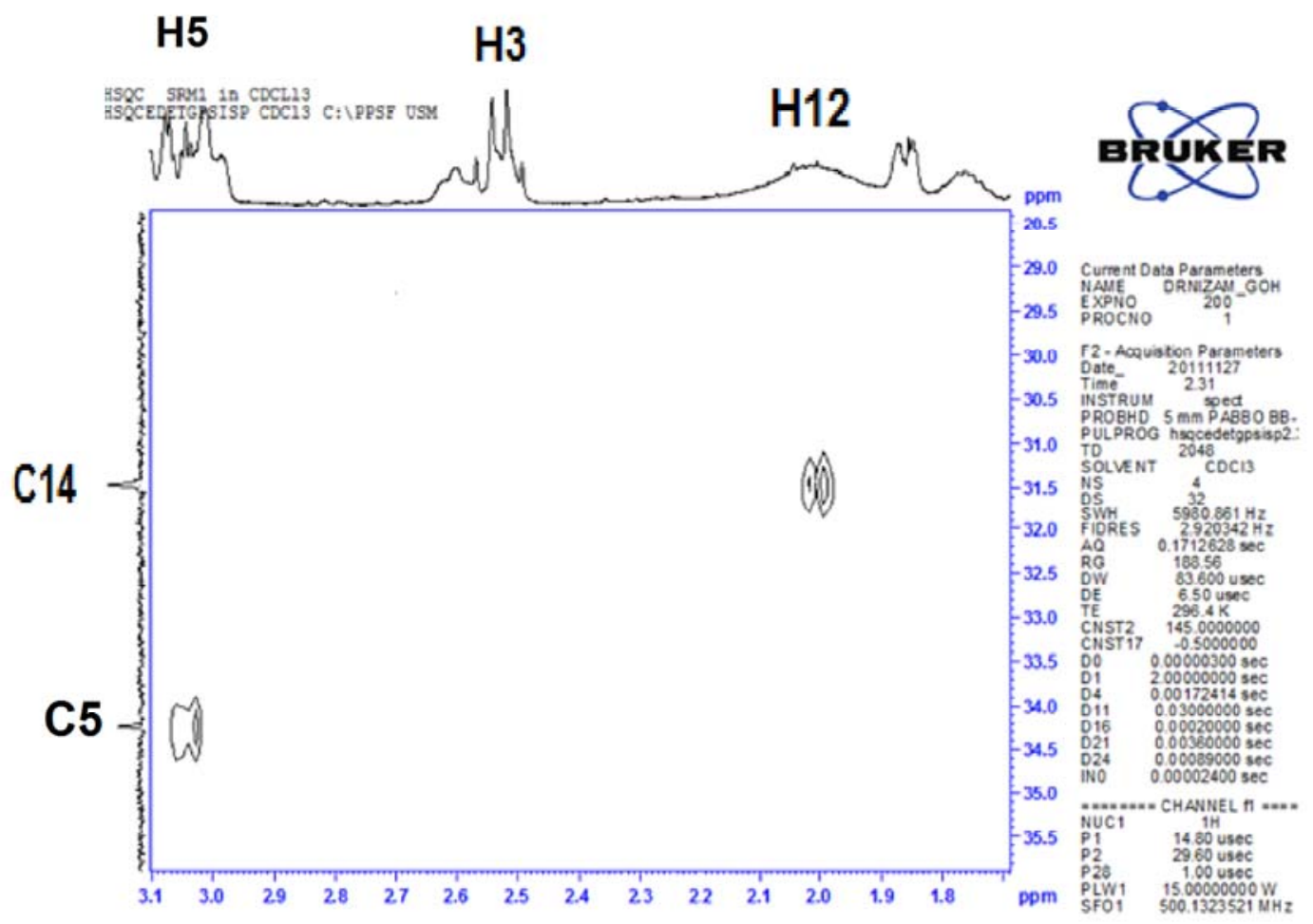

19. HSQC-Aliphatic Expanded Region

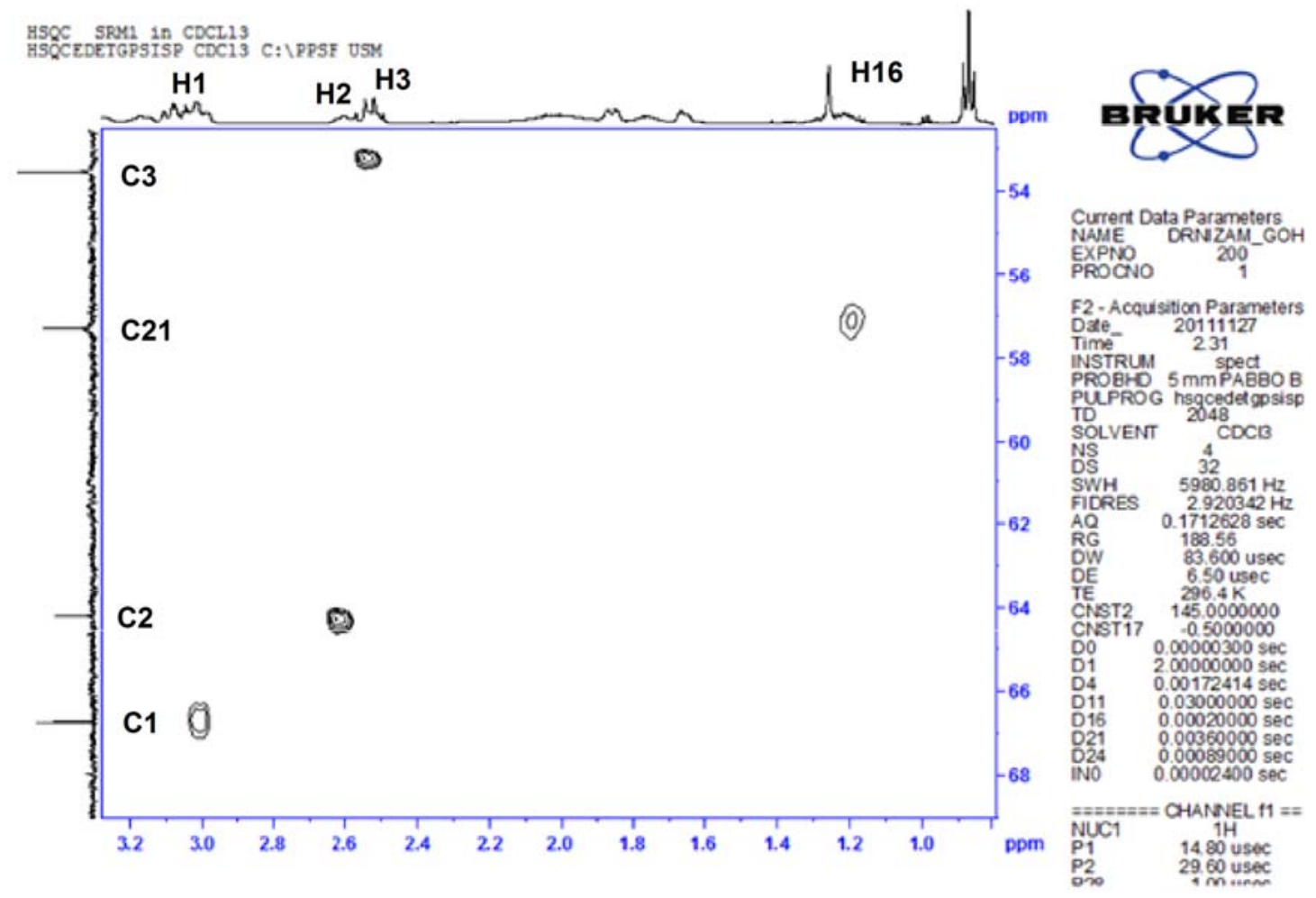


20. HSQC-Full Spectrum

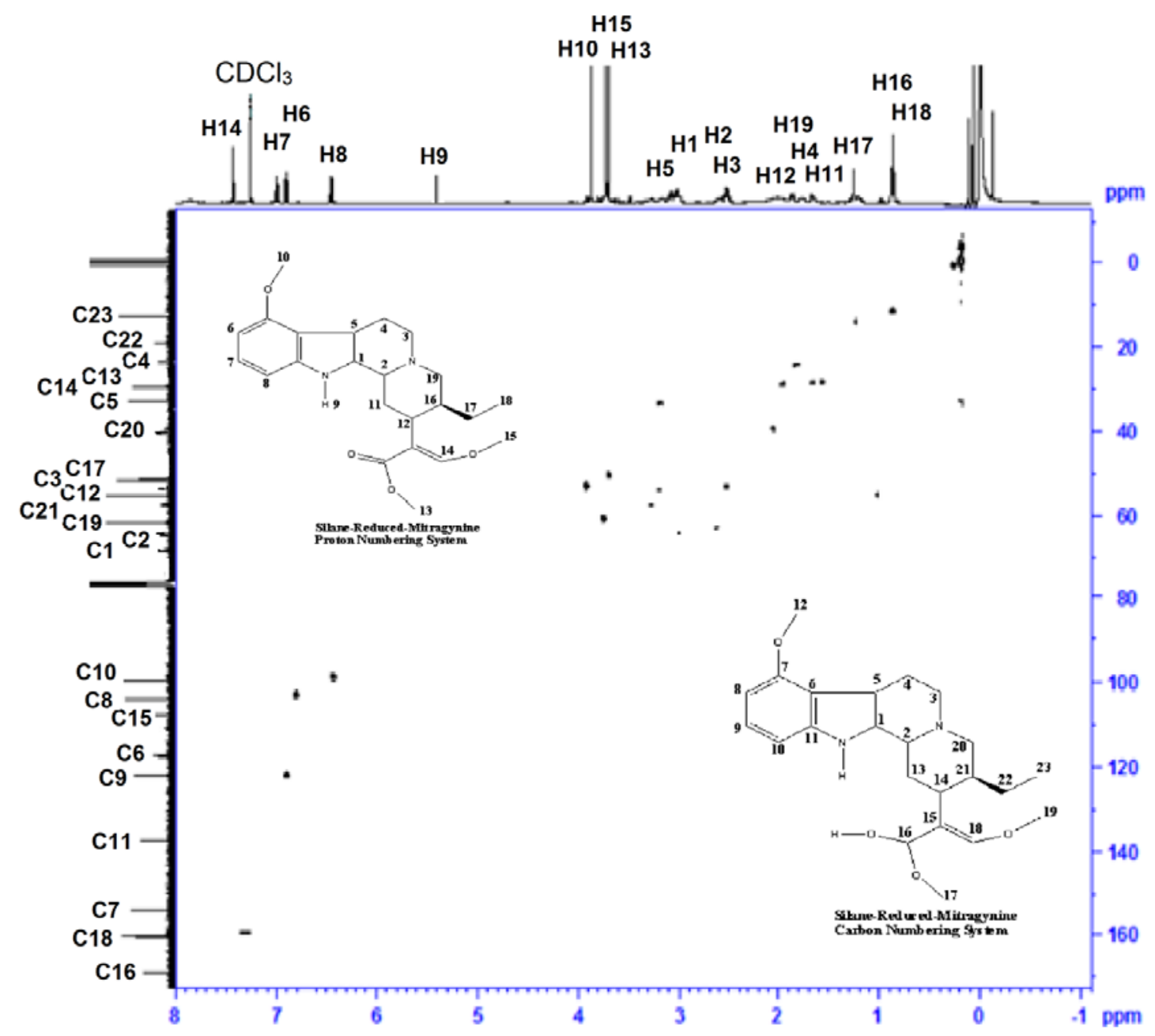


21 HSQC-Aromatic Expanded Region

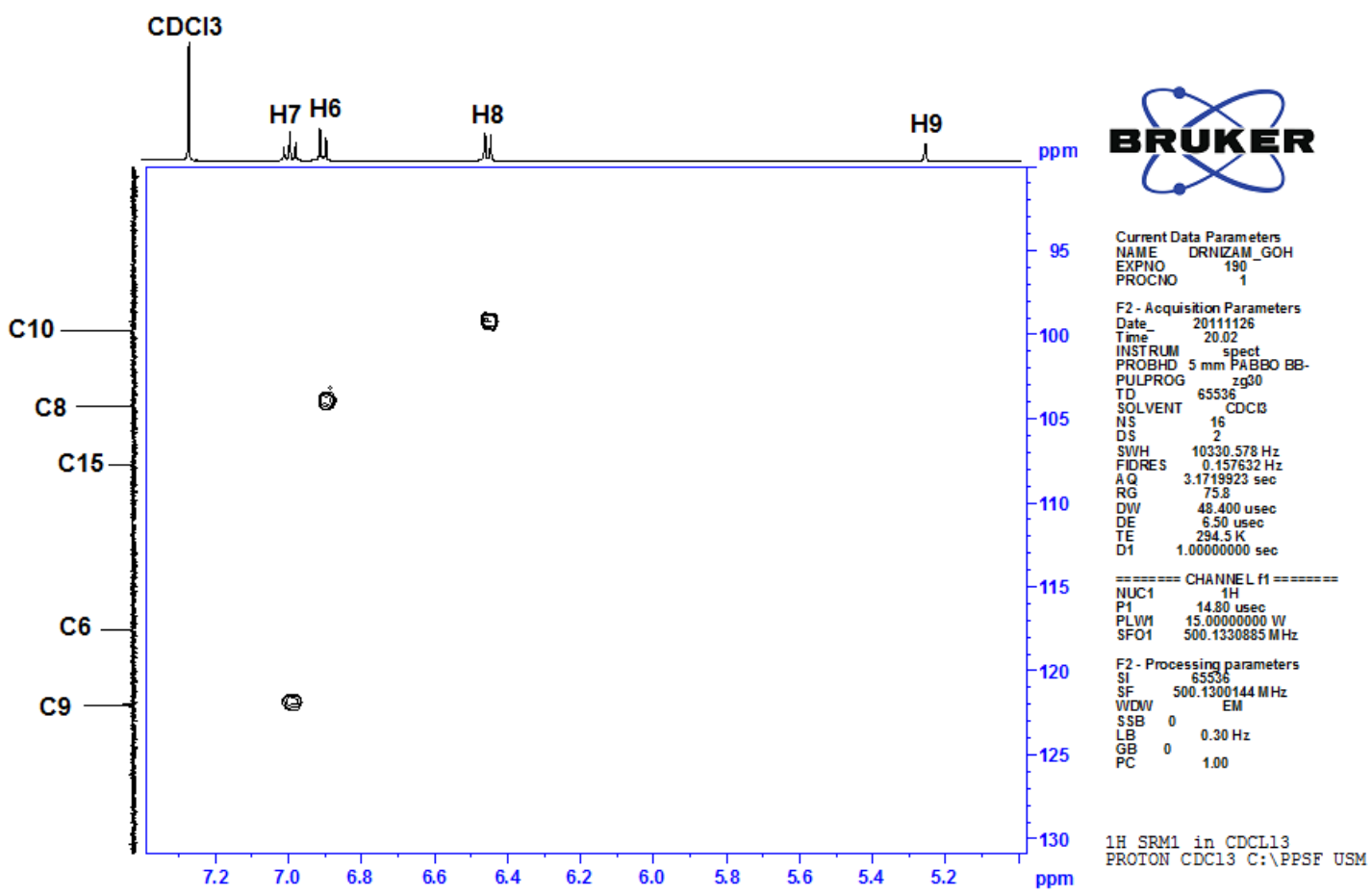

22. DQF-Cosy-Aromatic Expanded Region

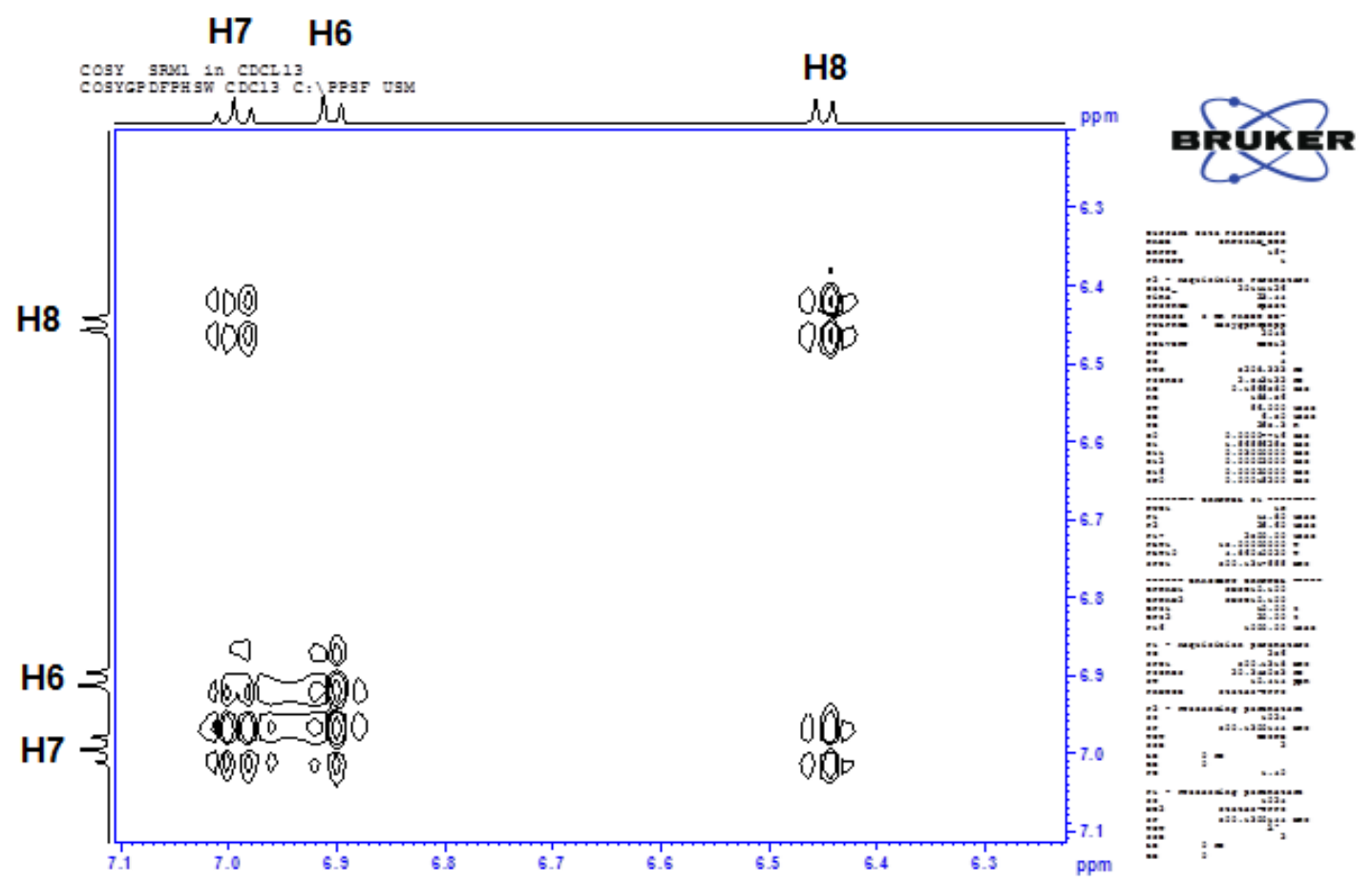


23. DQF-Cosy-Aliphatic Expanded Region

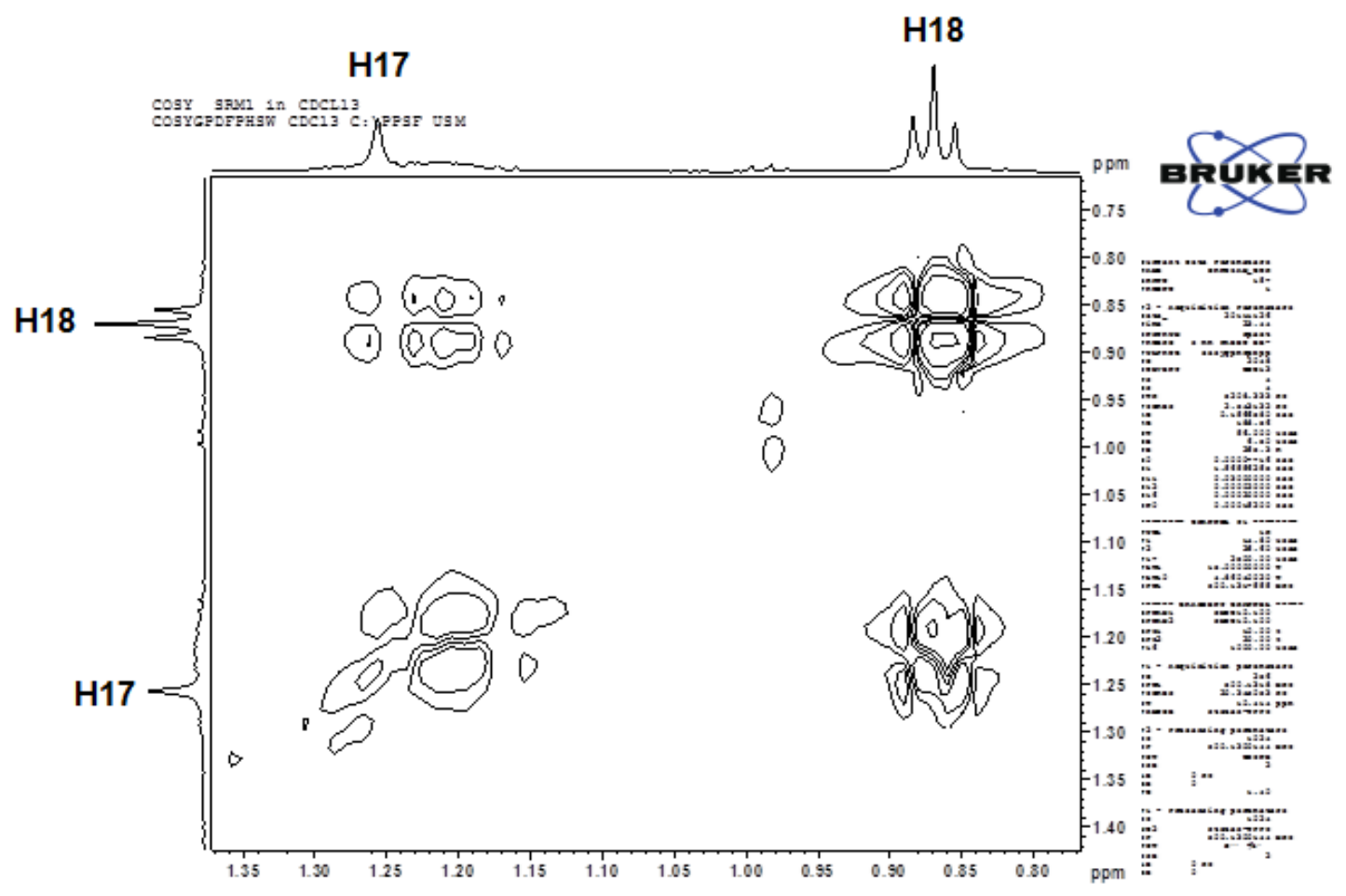

24. DQF-Cosy-Aliphatic Expanded Region

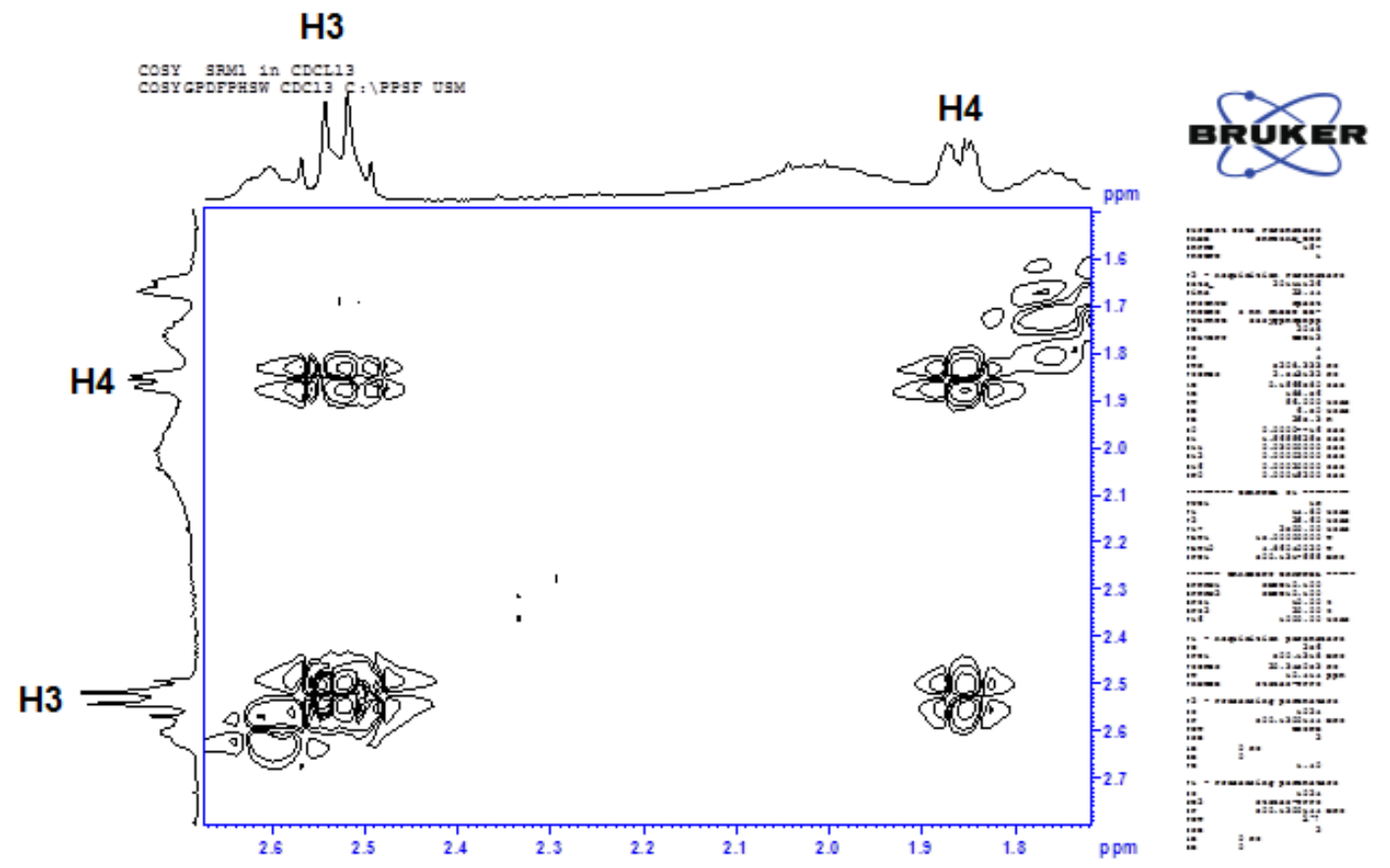


25. DQF-Cosy-Aliphatic Expanded Region

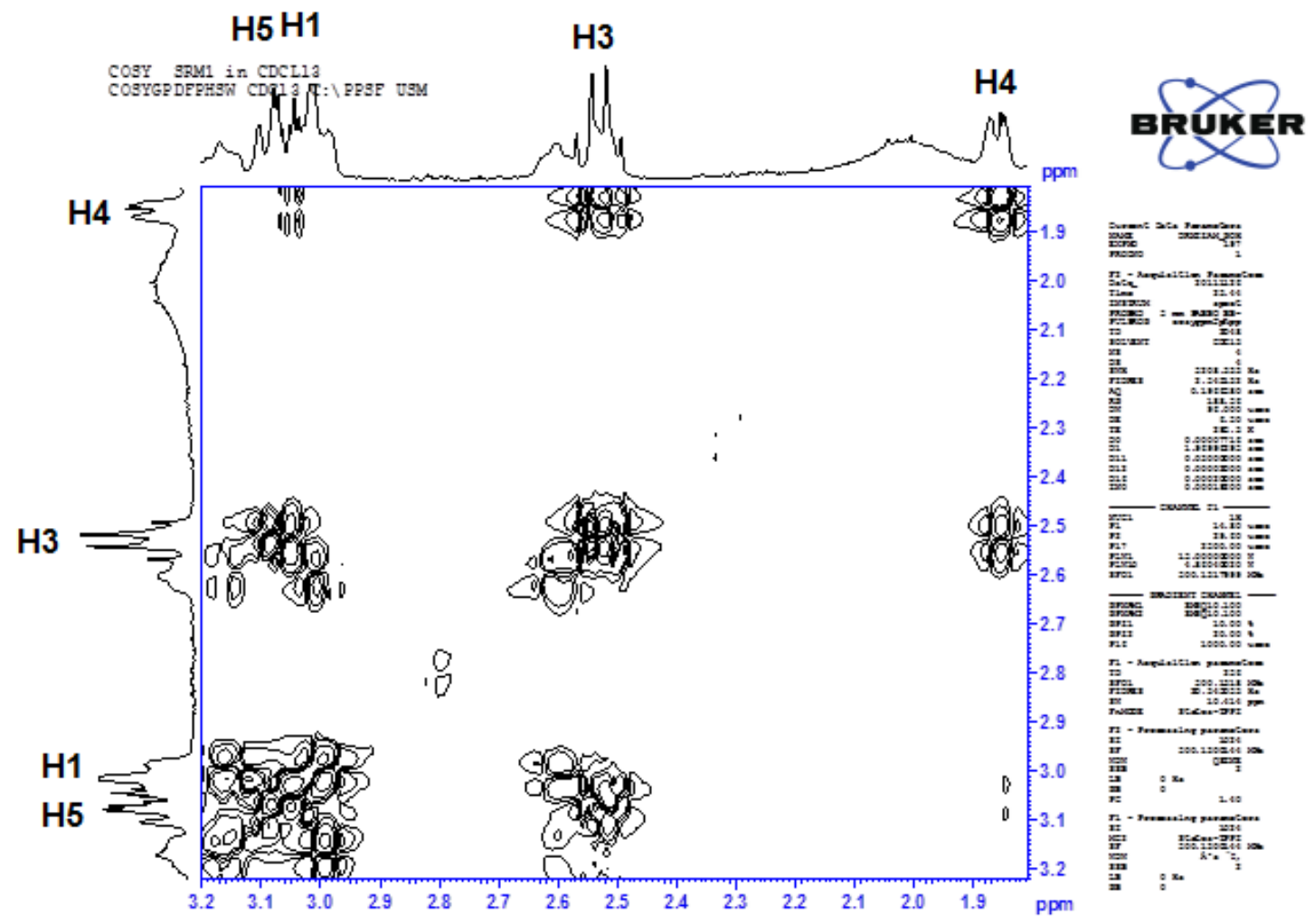

26. DQF-Cosy-Aliphatic Expanded Region
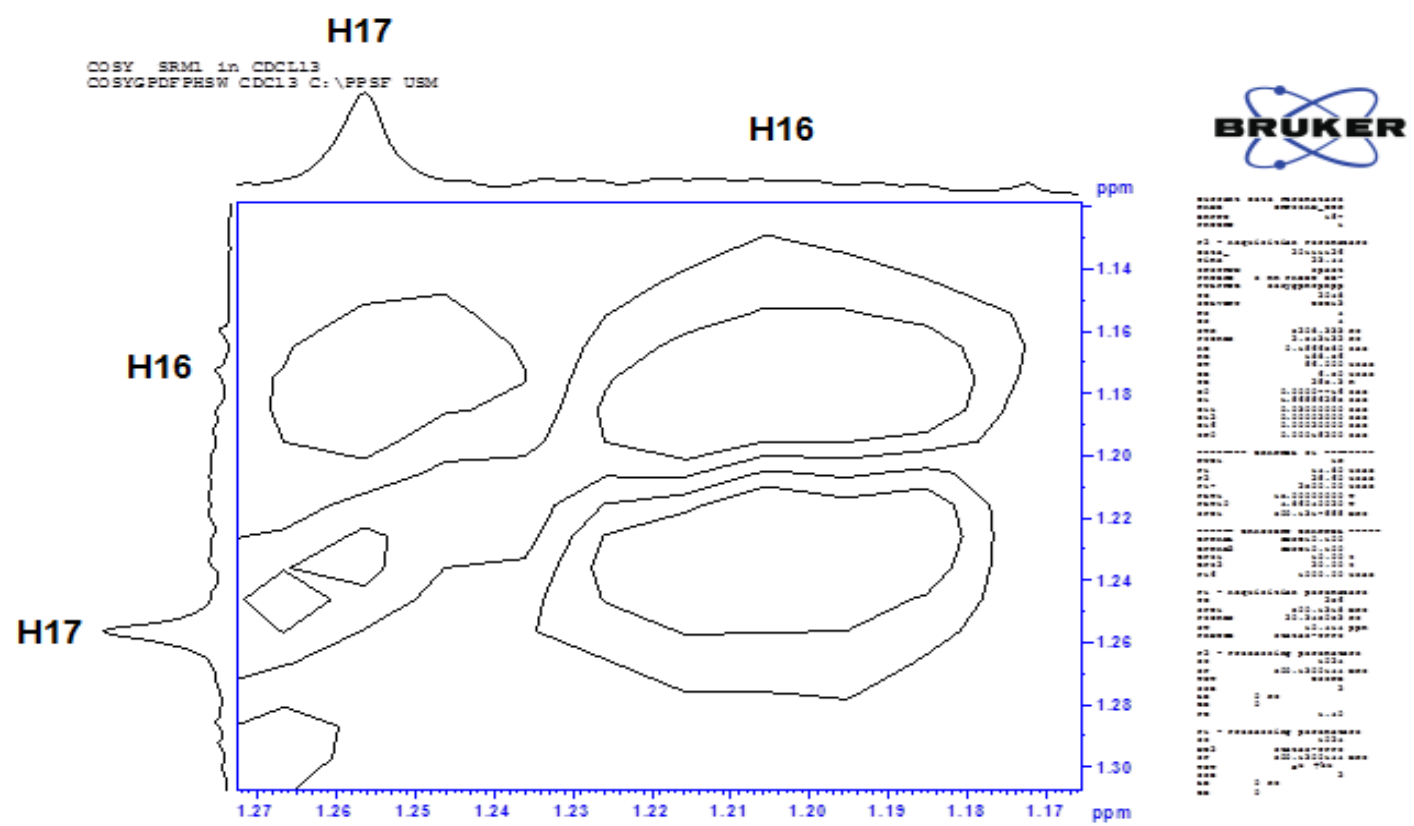
27. DQF-Cosy-Aliphatic Expanded Region

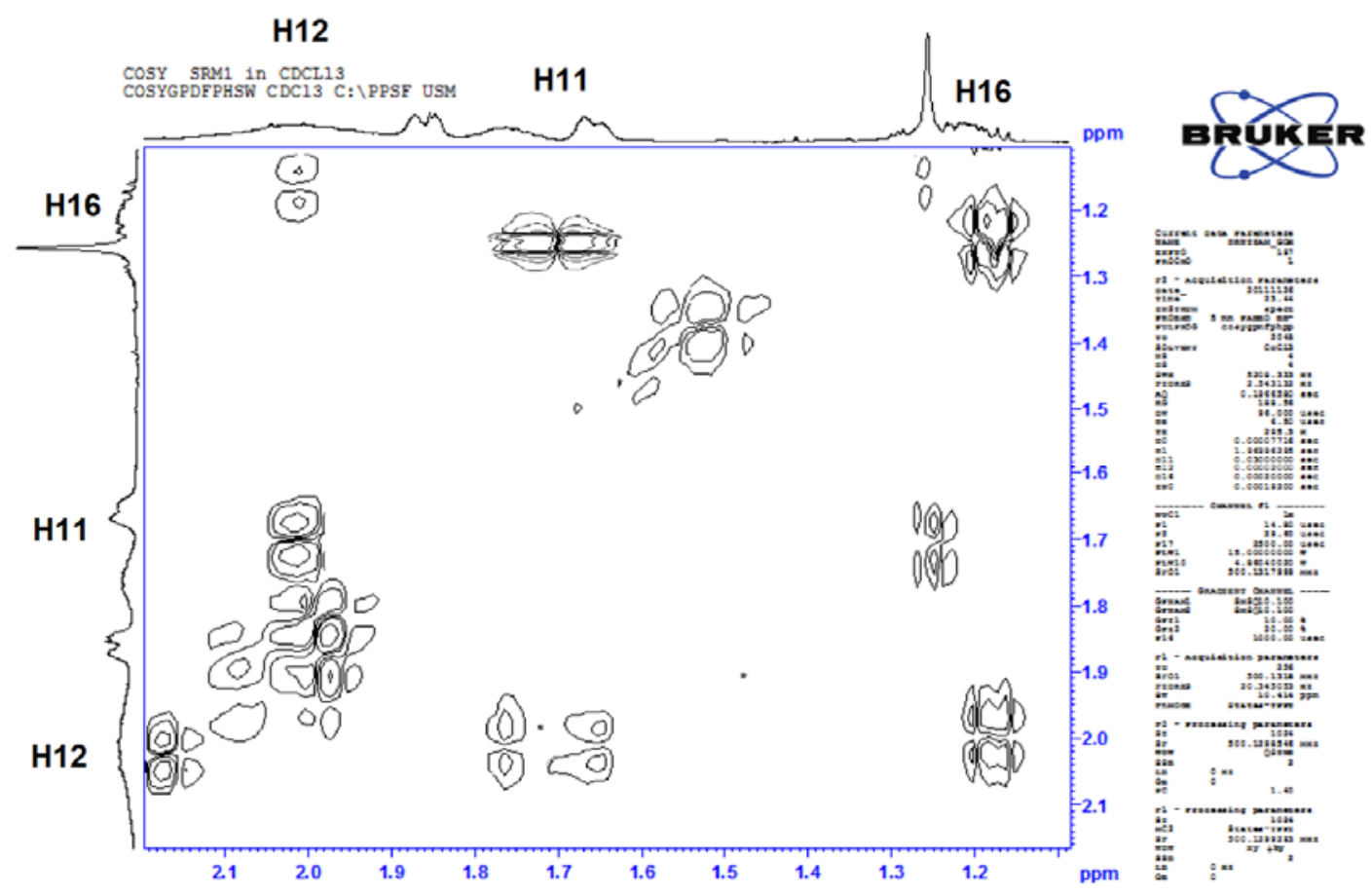

28 DQF-Cosy-Expanded Aliphatic Region

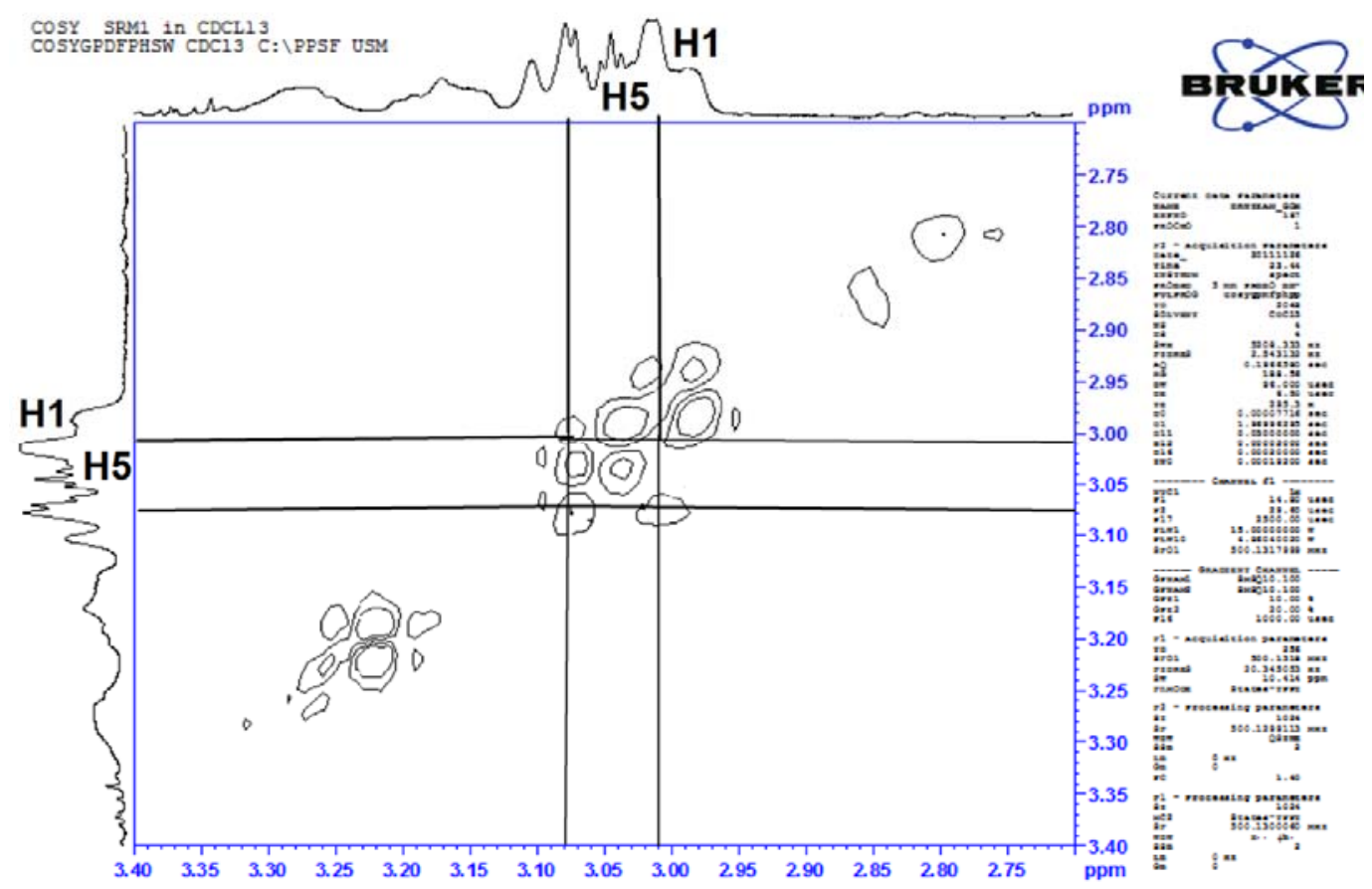


29 DQF-Cosy-Full spectrum

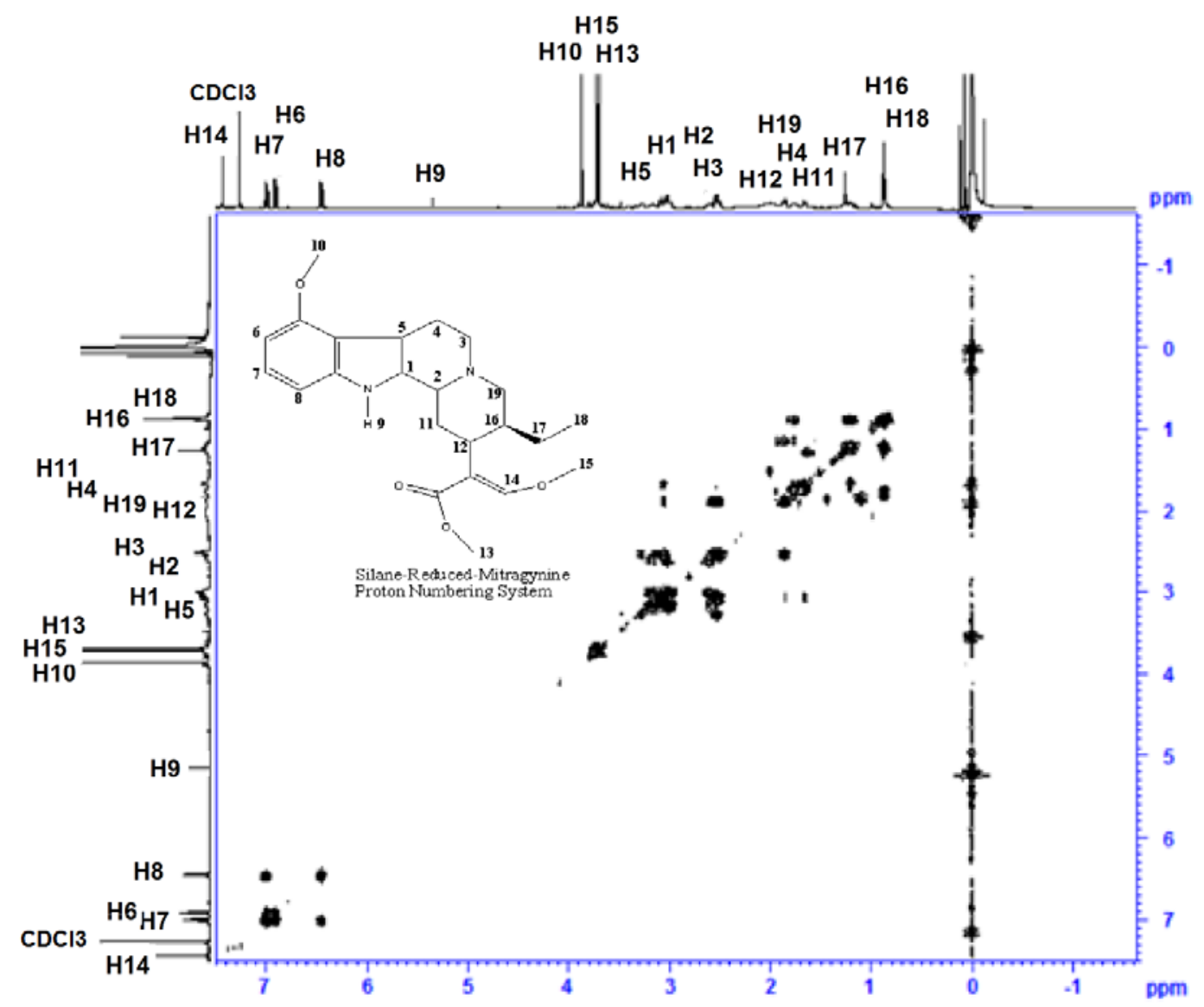

30. Tocsy-Expanded Aromatic Region

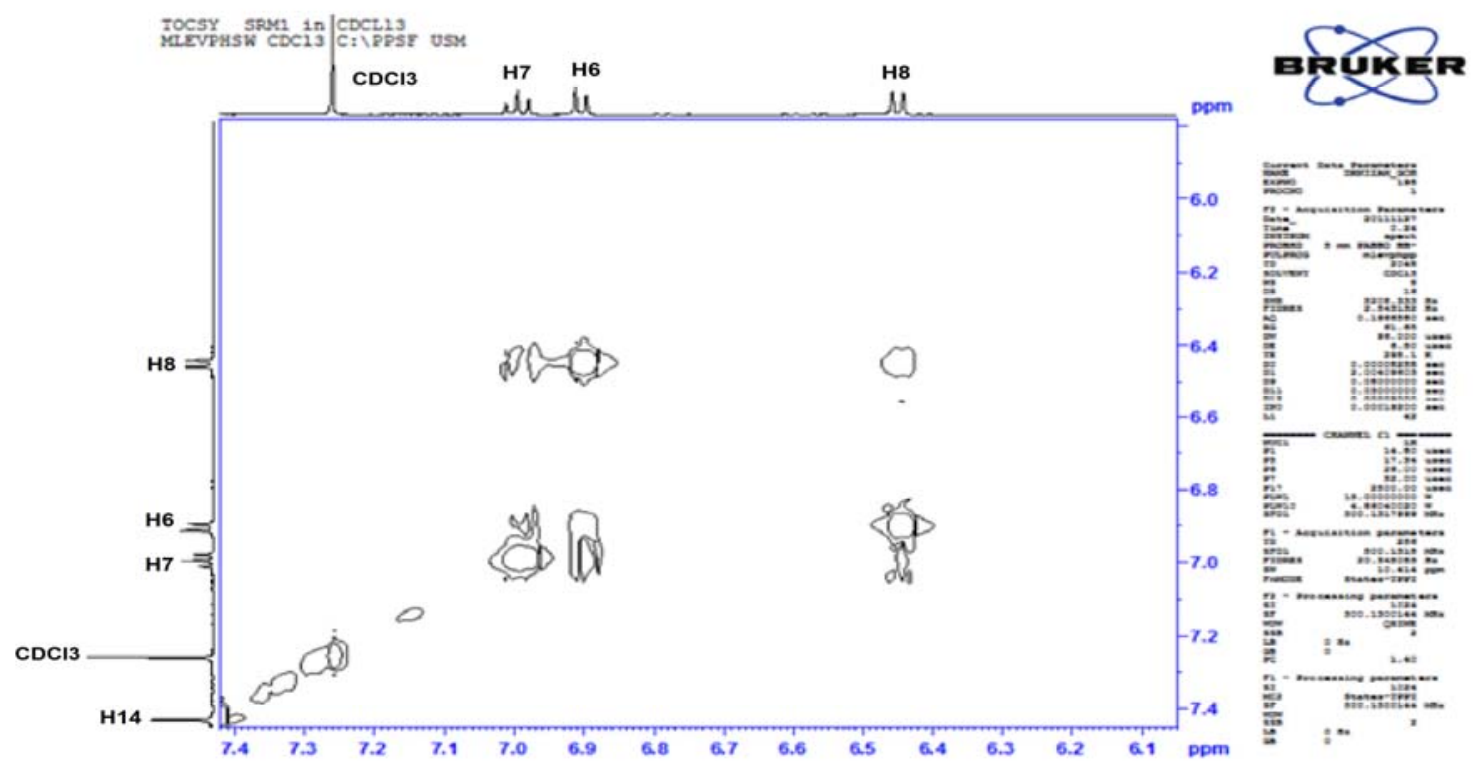


31. Tocsy-Expanded Aliphatic Region

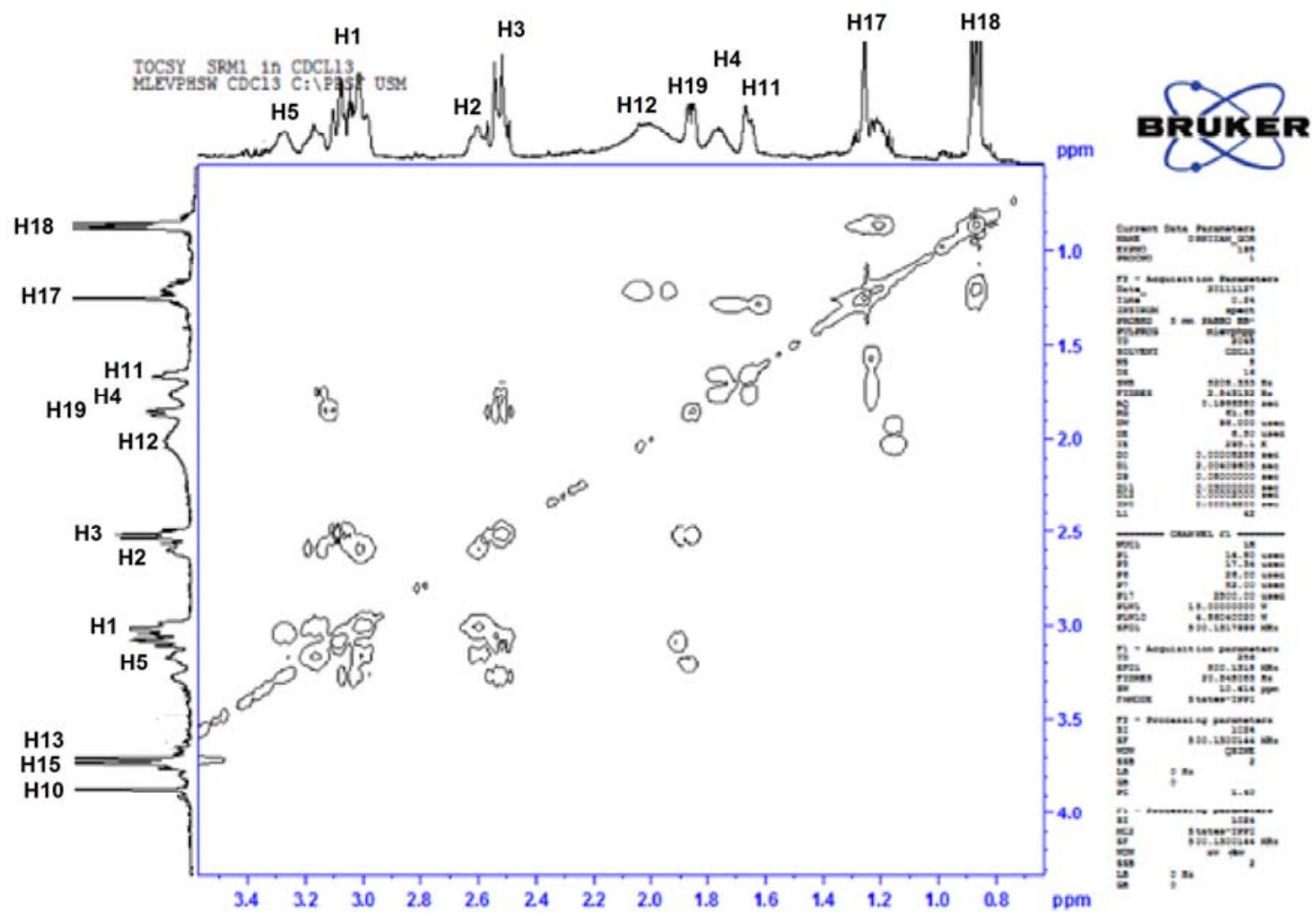

32 HMBC-Full spectrum

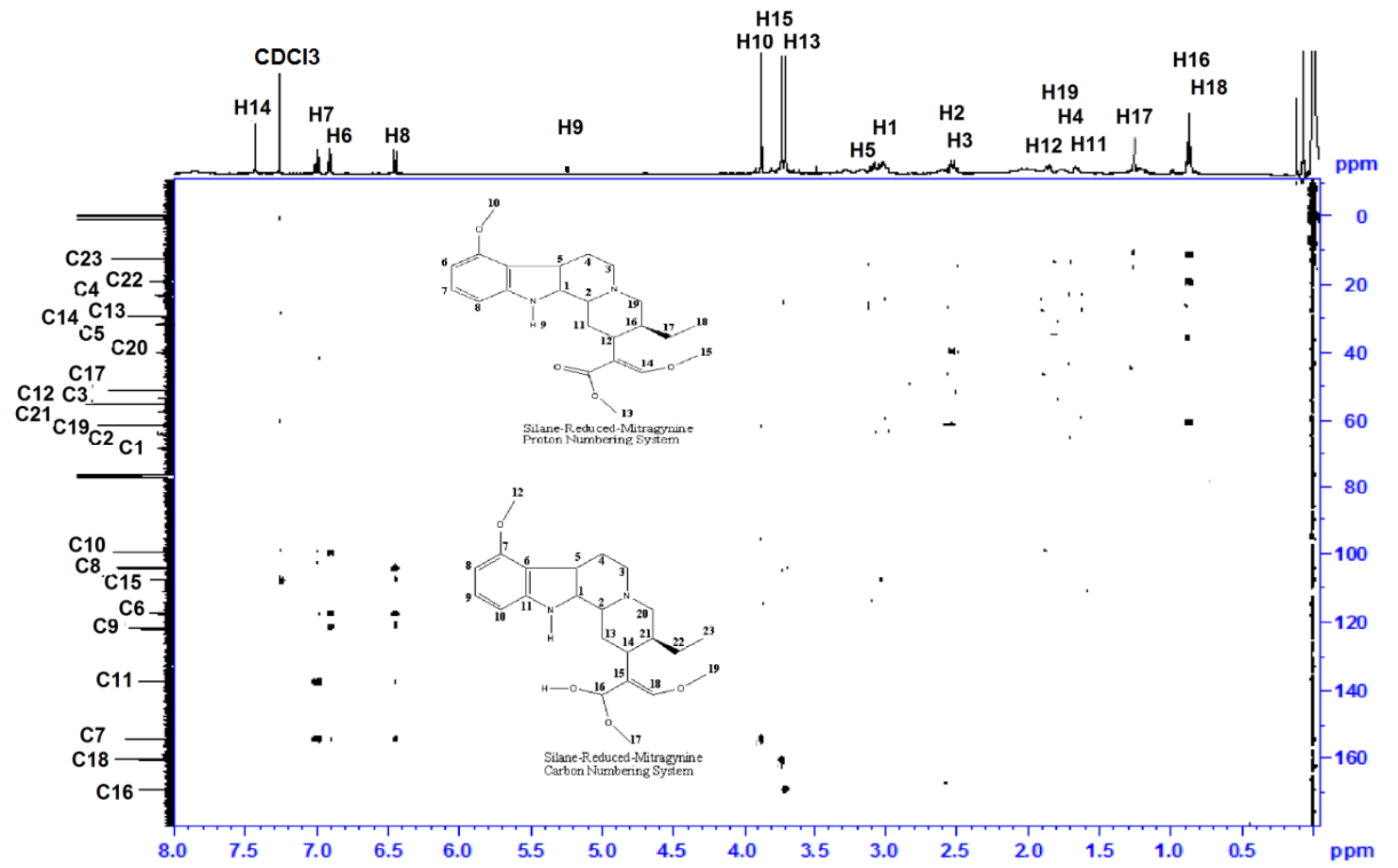


33 NOESY-Full spectrum

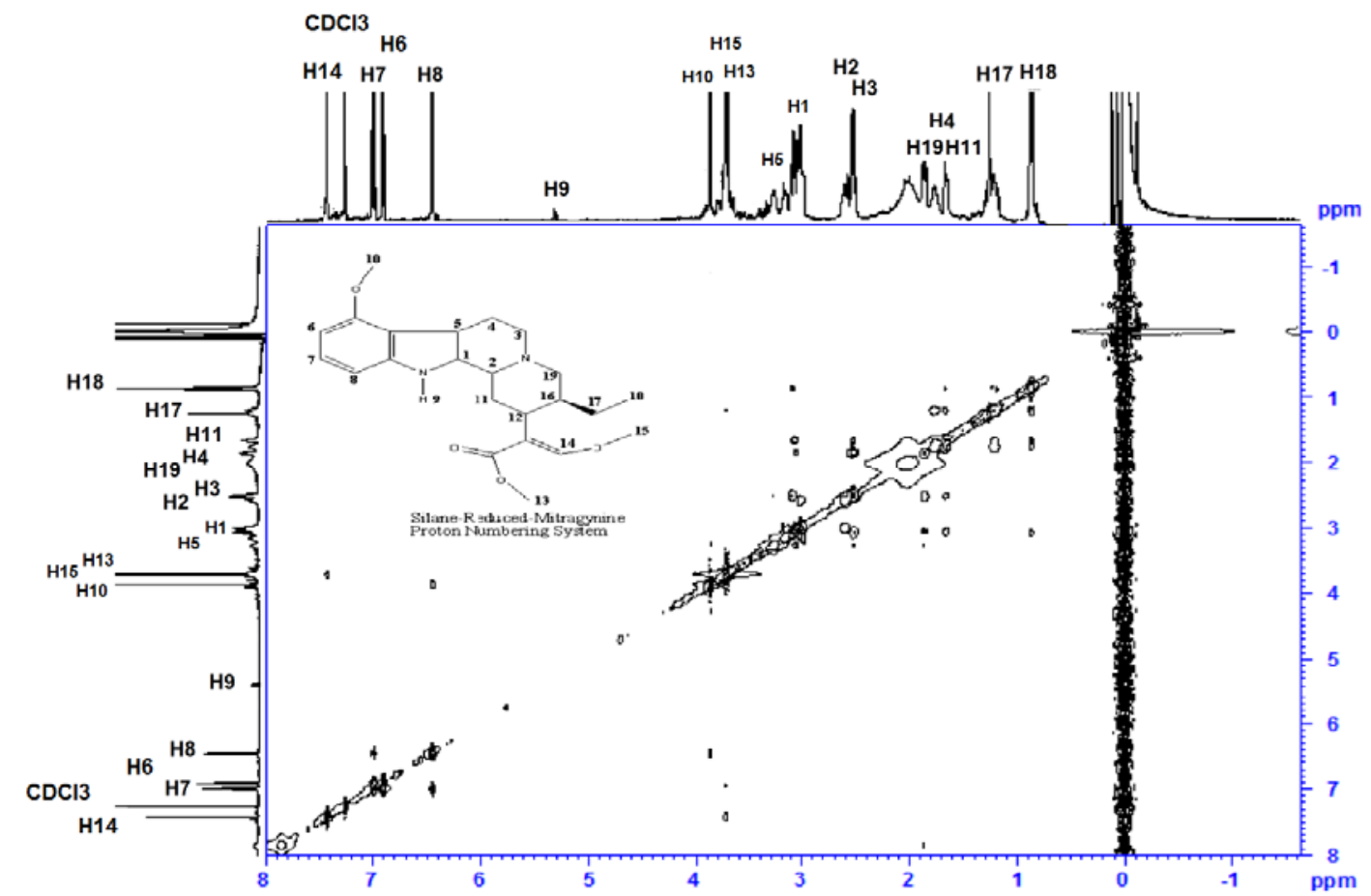

Figure 34 NOESY-Aliphatic expansion region

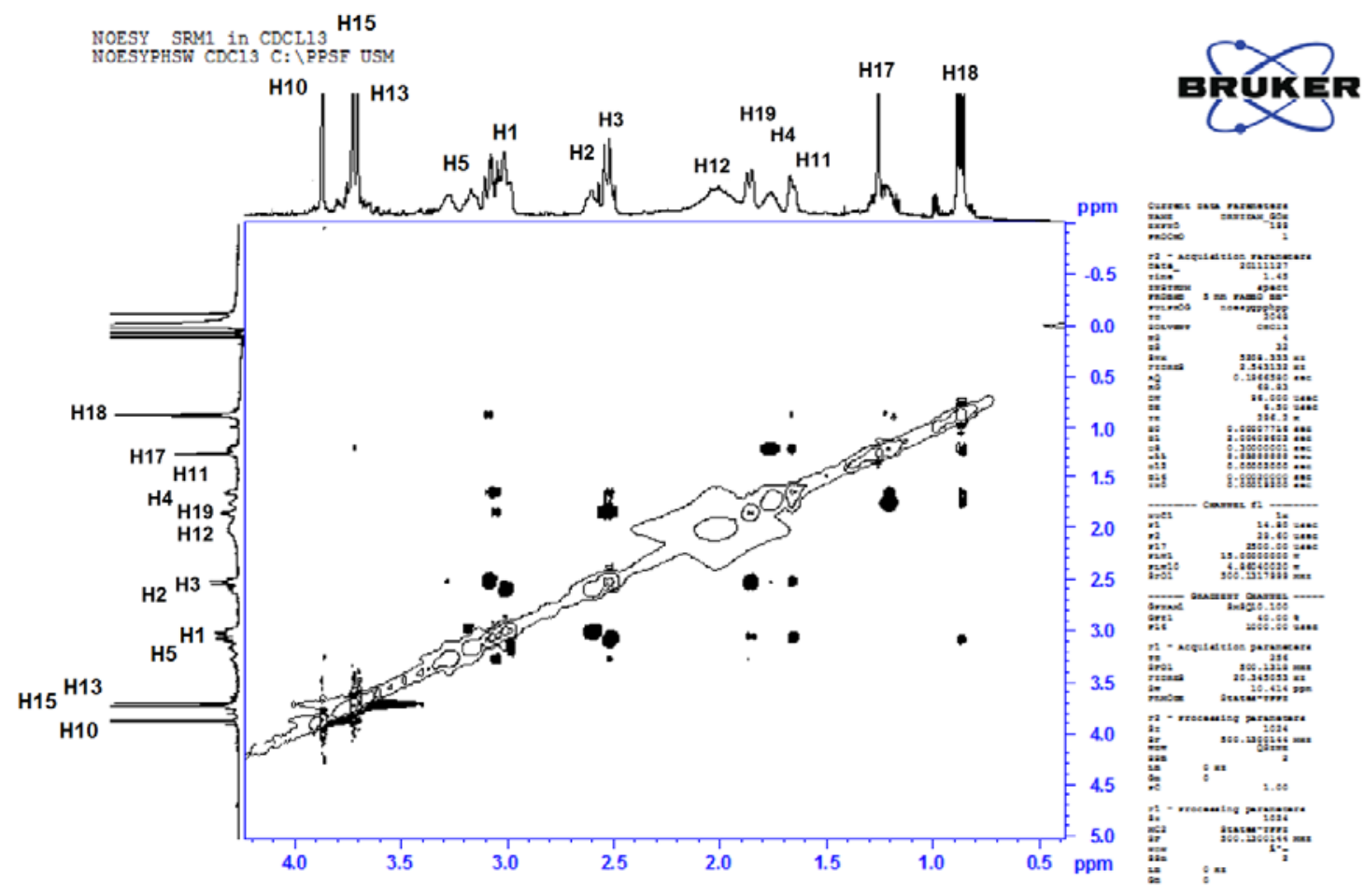

g 
35 CD Spectra

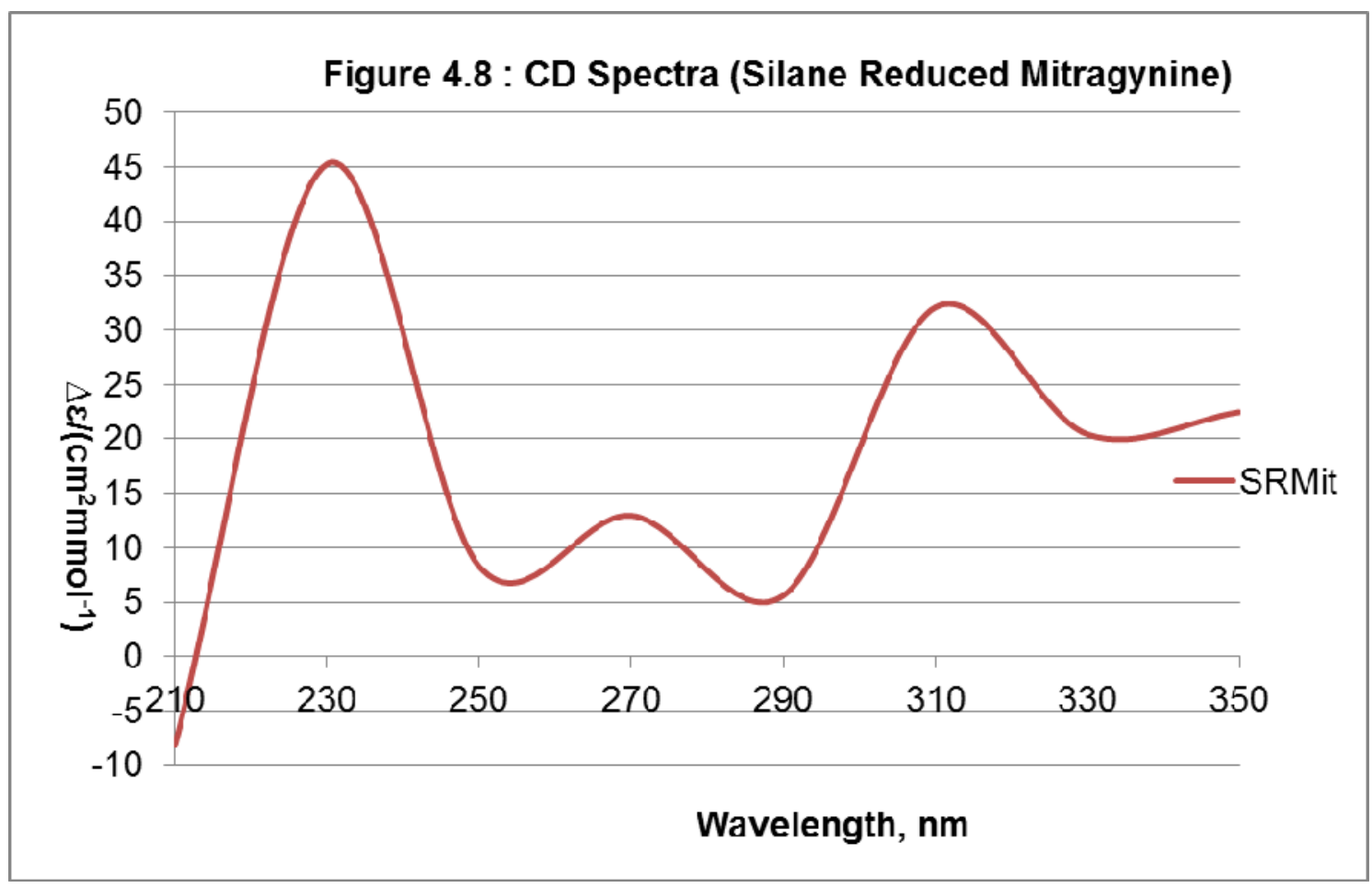




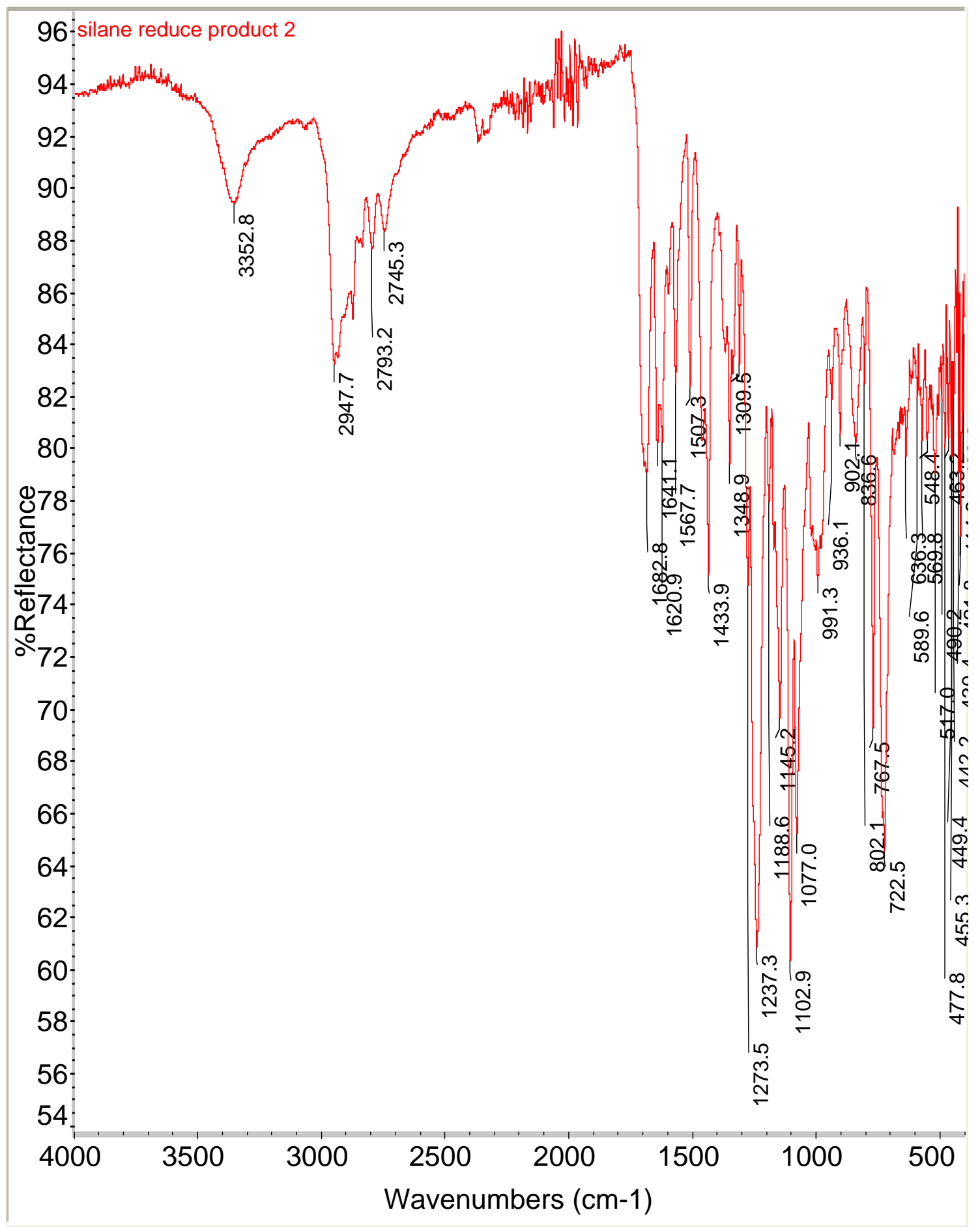


Spectrum Peak Pick Report

04/12/2011 08:12:41 PM

Data Set: RawData - C:IUsersIPenggunalDesktoplsrmit.spc $0.125 \mathrm{~g} / 1$

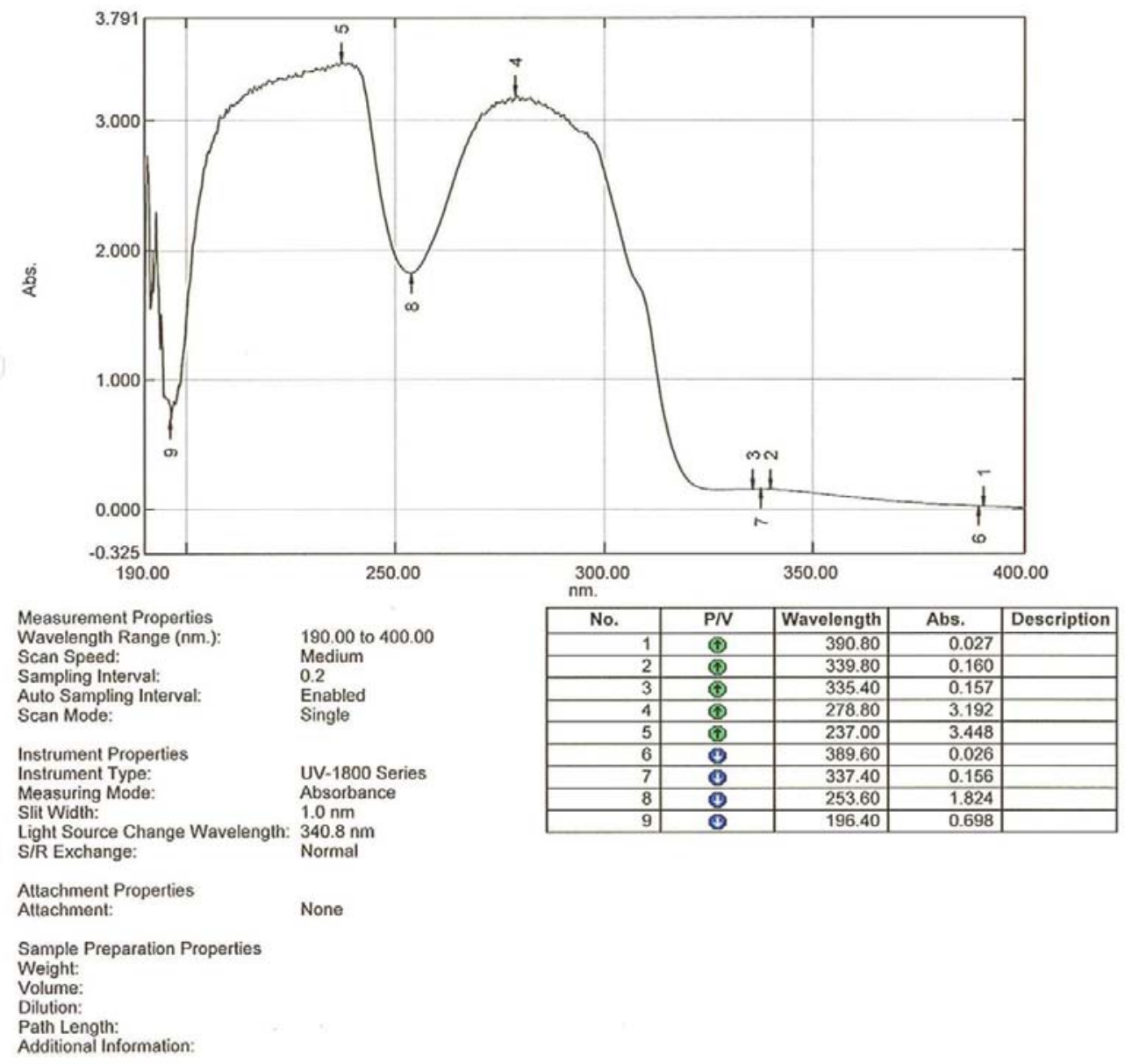

Page $1 / 1$ 
Sup 1 HPTLC fingerprint of the silane reduced mitragynine and the impurity / side product

\begin{tabular}{|c|c|c|}
\hline \multirow[t]{2}{*}{ Parameters } & \multicolumn{2}{|c|}{ TLC conditions / results } \\
\hline & Silane Reduced Mitragynine & Impurity / Side Product \\
\hline Quantity sample applied & $2 \mathrm{ul}$ & $2 \mathrm{ul}$ \\
\hline \multirow[t]{3}{*}{ Detector } & $12 \mathrm{~W}$ VL. 6.1C UV detector & $12 \mathrm{~W}$ VL. 6.1C UV detector \\
\hline & $6 \mathrm{~W}: 254 \mathrm{~nm}$ & $6 \mathrm{~W}: 254 \mathrm{~nm}$ \\
\hline & $6 \mathrm{~W}: 365 \mathrm{~nm}$ & $6 \mathrm{~W}: 365 \mathrm{~nm}$ \\
\hline Stationary Phase & Silica Gel $60 G_{254}$ & Silica Gel $60 G_{254}$ \\
\hline Mobile Phase & Hexane : Ethyl acetate $(92: 8)$ & Hexane : Ethyl acetate $(92: 8)$ \\
\hline Applied Concentration, $\mathrm{mg} / \mathrm{ml}$ & 0.05 & 0.05 \\
\hline Elution Length, $\mathrm{cm}$ & $8.0-10.0$ & $8.0-10.0$ \\
\hline $\mathrm{hR}_{\mathrm{f}, \mathrm{cm}}$ & 52.0 & 90.0 \\
\hline \multicolumn{3}{|l|}{ Visual appearance of Band: } \\
\hline White light & Yellowish & Yellowish \\
\hline UV 365 & Yellowish & Yellowish \\
\hline UV 254 & Brownish & Brownish \\
\hline Iodine & Yellowish brown & Yellowish brown \\
\hline Dragendorff reagent & Orange & Orange \\
\hline
\end{tabular}


Sup. 2 Summary of 1-D NMR data for silane reduced Mitragynine

\begin{tabular}{|c|c|c|c|c|c|c|c|}
\hline \multirow{2}{*}{$\begin{array}{c}\text { Hydrogen } \\
\text { No. }\end{array}$} & \multirow{2}{*}{$\begin{array}{c}\text { Carbon } \\
\text { No. }\end{array}$} & \multicolumn{6}{|c|}{ Chemical Shift, $\delta$, ppm } \\
\hline & & ${ }^{1} \mathrm{H}$ & ${ }^{13} \mathrm{C}$ & $\mathrm{CH}_{3}$ & $\mathrm{CH}_{2}$ & $\mathrm{CH}$ & $\mathrm{C}$ \\
\hline $\mathrm{H}-18$ & $\mathrm{C}-23$ & 0.87 & 12.81 & 12.81 & & & \\
\hline $\mathrm{H}-17$ & $\mathrm{C}-22$ & $1.26,1.29$ & 19.25 & & 19.25 & & \\
\hline $\mathrm{H}-4$ & C-4 & $1.86,1.87$ & 23.55 & & 23.55 & & \\
\hline $\mathrm{H}-11$ & $\mathrm{C}-13$ & $1.67,1.77$ & 29.74 & & 29.74 & & \\
\hline $\mathrm{H}-12$ & C-14 & 2.00 & 31.98 & & & 31.98 & \\
\hline $\mathrm{H}-5$ & C-5 & 3.08 & 34.32 & & & 34.32 & \\
\hline H-19 & $\mathrm{C}-20$ & $1.97,2.04$ & 40.36 & & 40.36 & & \\
\hline $\mathrm{H}-13$ & $\mathrm{C}-17$ & 3.70 & 51.36 & 51.36 & & & \\
\hline $\mathrm{H}-3$ & $\mathrm{C}-3$ & $2.52,2.54$ & 53.55 & & 53.55 & & \\
\hline $\mathrm{H}-10$ & $\mathrm{C}-12$ & 3.87 & 55.30 & 55.30 & & & \\
\hline H-16 & $\mathrm{C}-21$ & 1.19 & 57.33 & & & 57.33 & \\
\hline H-1 & $\mathrm{C}-1$ & 3.02 & 66.82 & & & 66.82 & \\
\hline $\mathrm{H}-15$ & $\mathrm{C}-19$ & 3.73 & 61.59 & 61.59 & & & \\
\hline $\mathrm{H}-2$ & $\mathrm{C}-2$ & 2.60 & 64.22 & & & 64.22 & \\
\hline $\mathrm{H}-8$ & $\mathrm{C}-10$ & 6.46 & 99.78 & & & 99.78 & \\
\hline H-6 & C-8 & 6.91 & 104.25 & & & 104.25 & \\
\hline- & $C-15$ & - & 111.25 & & & & 111.25 \\
\hline- & C-6 & - & 117.55 & & & & 117.55 \\
\hline $\mathrm{H}-7$ & C-9 & 7.00 & 122.03 & & & 122.03 & \\
\hline- & C-11 & - & 137.34 & & & & 137.34 \\
\hline- & C-7 & - & 154.53 & & & & 154.53 \\
\hline H-14 & C-18 & 7.43 & 160.60 & & & 160.60 & \\
\hline
\end{tabular}


$\begin{array}{llll}- & \mathrm{C}-16 & - & 169.14\end{array}$

169.14

\begin{tabular}{cccccccc} 
H-9 & & 5.23 & - & & & & \\
$(\mathrm{N}-\mathrm{H})$ & & & & & & \\
\hline Total & Total & 32 & 23 & 4 & 5 & 9 & 5 \\
\hline
\end{tabular}

Sup 3 Summary of 2D-NMR data for silane reduced mitragynine

\begin{tabular}{|c|c|c|c|c|c|}
\hline \multirow{2}{*}{$\begin{array}{c}\text { Hydrogen } \\
\text { No. }\end{array}$} & \multirow{2}{*}{$\begin{array}{c}\text { Carbon } \\
\text { No. }\end{array}$} & \multicolumn{4}{|c|}{ Chemical Shift, $\delta$} \\
\hline & & $\begin{array}{l}\text { HSQC } \\
\text { C-H }\end{array}$ & $\begin{array}{l}\text { HMBC } \\
\text { C-H' }\end{array}$ & $\begin{array}{c}\text { DQF COSY } \\
\text { H-H' }\end{array}$ & $\begin{array}{c}\text { TOCSY } \\
\text { H-H' }\end{array}$ \\
\hline H-18 & $\mathrm{C}-23$ & $\begin{array}{l}\text { H18 (0.87)- } \\
\text { C23 (12.81) }\end{array}$ & $\begin{array}{c}\text { H18 (0.87)- } \\
\text { C23 (12.81) } \\
\text { H17 }(1.26,1.29)- \\
\text { C22 (19.25) } \\
\text { H16(1.19)- } \\
\text { C21(57.33) } \\
\text { H19(1.97,2.04)- } \\
\text { C20(40.36) } \\
\text { H12(2.00)- } \\
\text { C14(31.98) }\end{array}$ & $\begin{array}{c}\text { H18(0.87)- } \\
\text { H17(1.26,1.29) }\end{array}$ & $\begin{array}{c}\text { H18(0.87)- } \\
\text { H17(1.26,1.29) }\end{array}$ \\
\hline $\mathrm{H}-17$ & $\mathrm{C}-22$ & $\begin{array}{l}\mathrm{H} 17(1.26,1.29)- \\
\quad \mathrm{C} 22(19.25)\end{array}$ & $\begin{array}{c}\text { H17 }(1.26,1.29)- \\
\text { C22 }(19.25) \\
\text { H18 (0.87)- } \\
\text { C23 }(12.81) \\
\text { H16(1.19)- } \\
\text { C21(57.33) } \\
\text { H19(1.97,2.04)- } \\
\text { C20(40.36) } \\
\text { H12(2.00)- } \\
\text { C14(31.98) }\end{array}$ & $\begin{array}{c}\mathrm{H} 17(1.26,1.29)- \\
\mathrm{H} 16(1.19) \\
\mathrm{H} 17(1.26,1.29)- \\
\mathrm{H} 18(0.87)\end{array}$ & $\begin{array}{l}\text { H17(1.26,1.29)- } \\
\text { H16(1.19) } \\
\text { H17(1.26,1.29)- } \\
\text { H18(0.87) }\end{array}$ \\
\hline $\mathrm{H}-4$ & $\mathrm{C}-4$ & $\begin{array}{c}\mathrm{H} 4(1.86,1.87)- \\
\mathrm{C} 4(23.55)\end{array}$ & $\begin{array}{c}\mathrm{H} 4(1.86,1.87)- \\
\mathrm{C} 4(23.55) \\
\mathrm{H} 3(2.52,2.54)- \\
\mathrm{C} 3(53.55) \\
\mathrm{H} 5(3.08)- \\
\text { C5(34.32) } \\
\text { H1 }(3.01)- \\
\text { C1 }(66.82\end{array}$ & $\begin{array}{c}\mathrm{H} 4(2.76,2.87)- \\
\mathrm{H} 3(3.09,3.32)\end{array}$ & $\begin{array}{c}\mathrm{H} 4(2.76,2.87)- \\
\mathrm{H} 3(3.09,3.32)\end{array}$ \\
\hline
\end{tabular}

H-11

$\mathrm{C}-1311(1.67,1.77)-$
$\mathrm{C} 13(29.74)$

H11(1.67, 1.77)- H11(1.67,1.77)- $\quad$ H11(1.67,1.77)-

C13(29.74) $\mathrm{H} 2(2.60)$

$\mathrm{H} 2(2.60)$

H12(2.00)- H11(1.67, 1.77)- H11(1.67, 1.77)- 


\begin{tabular}{|c|c|c|c|c|c|}
\hline & & & $\begin{array}{l}\mathrm{C} 14(31.98) \\
\mathrm{H} 2(2.60)- \\
\mathrm{C} 2(64.22) \\
\mathrm{H} 1(3.01)- \\
\mathrm{C} 1(66.82) \\
\mathrm{H} 16(1.19)- \\
\mathrm{C} 21(57.33)\end{array}$ & $\mathrm{H} 12(2.00)$ & $\mathrm{H} 12(2.00)$ \\
\hline $\mathrm{H}-12$ & $\mathrm{C}-14$ & $\begin{array}{l}\mathrm{H} 12(2.00)- \\
\mathrm{C} 14(31.98)\end{array}$ & $\begin{array}{l}\mathrm{H} 12(2.00)- \\
\mathrm{C} 14(31.98)\end{array}$ & $\begin{array}{c}\text { H12(2.00)- } \\
\text { H11 }(1.67,1.77) \\
\text { H12(2.00)- } \\
\text { H16(1.19) }\end{array}$ & $\begin{array}{c}\mathrm{H} 12(2.00)-\mathrm{H} 11(1.67, \\
1.77) \\
\mathrm{H} 12(2.00)-\mathrm{H} 16(1.19)\end{array}$ \\
\hline H-5 & $\mathrm{C}-5$ & $\mathrm{H} 5(3.08)-\mathrm{C} 5(34.32)$ & $\begin{array}{l}\mathrm{H} 5(3.08)- \\
\mathrm{C} 5(34.32)\end{array}$ & $\mathrm{H} 7(7.00)-\mathrm{H} 6(6.91)$ & H7(7.00)-H6(6.91) \\
\hline H-19 & $\mathrm{C}-20$ & $\begin{array}{c}\mathrm{H} 19(1.97,2.04)- \\
\mathrm{C} 20(40.36)\end{array}$ & $\begin{array}{l}\mathrm{H} 19(1.97,2.04)- \\
\quad \mathrm{C} 20(40.36)\end{array}$ & $\begin{array}{c}\mathrm{H} 19(1.97,2.04)- \\
\mathrm{H} 16(1.19)\end{array}$ & $\begin{array}{c}\text { H19(1.97,2.04)- } \\
\text { H16(1.19) }\end{array}$ \\
\hline $\mathrm{H}-13$ & $\mathrm{C}-17$ & $\begin{array}{l}\mathrm{H} 13(3.70)- \\
\mathrm{C} 17(51.36)\end{array}$ & $\begin{array}{l}\text { H13(3.70)- } \\
\text { C17(51.36) }\end{array}$ & - & - \\
\hline $\mathrm{H}-3$ & $\mathrm{C}-3$ & $\begin{array}{l}\mathrm{H} 3(2.52,2.54)- \\
\quad \mathrm{C} 3(53.55)\end{array}$ & $\begin{array}{l}\mathrm{H} 3(2.52,2.54)- \\
\mathrm{C} 3(53.55)\end{array}$ & $\begin{array}{l}\mathrm{H} 3(3.09,3.32)- \\
\mathrm{H} 4(2.76,2.87)\end{array}$ & $\begin{array}{l}\mathrm{H} 3(3.09,3.32)- \\
\mathrm{H} 4(2.76,2.87)\end{array}$ \\
\hline $\mathrm{H}-10$ & $\mathrm{C}-12$ & $\begin{array}{l}\mathrm{H} 10(3.87)- \\
\mathrm{C} 12(55.30)\end{array}$ & $\begin{array}{l}\mathrm{H} 10(3.87)- \\
\mathrm{C} 12(55.30)\end{array}$ & - & - \\
\hline H-16 & $\mathrm{C}-21$ & $\begin{array}{l}\text { H16(1.19)- } \\
\text { C21(57.33) }\end{array}$ & $\begin{array}{l}\text { H16(1.19)- } \\
\text { C21(57.33) }\end{array}$ & $\begin{array}{c}\text { H16(1.19)- } \\
\text { H12(2.00) } \\
\text { H16(1.19)- } \\
\text { H17(1.26,1.29) } \\
\text { H16(1.19)- } \\
\text { H19(1.97,2.04) }\end{array}$ & $\begin{array}{c}\text { H16(1.19)- } \\
\text { H12(2.00) } \\
\text { H16(1.19)- } \\
\text { H17(1.26,1.29) } \\
\text { H16(1.19)- } \\
\text { H19(1.97,2.04) }\end{array}$ \\
\hline H-1 & $\mathrm{C}-1$ & $\begin{array}{l}\mathrm{H} 1(3.02)- \\
\mathrm{C} 1(66.82)\end{array}$ & & $\begin{array}{l}\mathrm{H} 1(3.02)-\mathrm{H} 5(3.08) \\
\mathrm{H} 1(3.02)-\mathrm{H} 2(2.60)\end{array}$ & $\begin{array}{l}\mathrm{H} 1(3.02)-\mathrm{H} 5(3.08) \\
\mathrm{H} 1(3.02)-\mathrm{H} 2(2.60)\end{array}$ \\
\hline H-15 & C-19 & $\begin{array}{l}\mathrm{H} 15(3.73)-\mathrm{H} \\
\mathrm{C} 19(61.59)\end{array}$ & $\begin{array}{l}\mathrm{H} 1(3.02)-\mathrm{C} 1(66.82) \\
\begin{array}{l}\mathrm{H} 15(3.73)- \\
\text { C19(61.59) }\end{array}\end{array}$ & - & - \\
\hline $\mathrm{H}-2$ & $\mathrm{C}-2$ & $\begin{array}{l}\mathrm{H} 2(2.60)- \\
\mathrm{C} 2(64.22)\end{array}$ & $\mathrm{H} 2(2.60)-\mathrm{C} 2(64.22)$ & $\mathrm{H} 2(2.60)-\mathrm{H} 1(3.01)$ & $\mathrm{H} 2(2.60)-\mathrm{H} 1(3.01)$ \\
\hline $\mathrm{H}-8$ & $\mathrm{C}-10$ & $\begin{array}{l}\mathrm{H} 8(6.46)- \\
\text { C10(99.78) }\end{array}$ & $\begin{array}{l}\mathrm{H} 8(6.46)- \\
\mathrm{C} 10(99.78)\end{array}$ & $\mathrm{H} 8(6.46)-\mathrm{H} 7(7.00)$ & $\mathrm{H} 8(6.46)-\mathrm{H} 7(7.00)$ \\
\hline H-6 & $\mathrm{C}-8$ & $\begin{array}{l}\mathrm{H} 6(6.91)- \\
\text { C8(104.25) }\end{array}$ & $\begin{array}{l}\mathrm{H} 6(6.91)- \\
\text { C8(104.25) }\end{array}$ & H6(6.91)-H7(7.00) & H6(6.91)-H7(7.00) \\
\hline- & C-15 & - & & - & - \\
\hline- & C-6 & - & & - & - \\
\hline $\mathrm{H}-7$ & $\mathrm{C}-9$ & $\begin{array}{l}\mathrm{H} 7(7.00)- \\
\mathrm{C} 9(122.03)\end{array}$ & $\begin{array}{l}\mathrm{H} 7(7.00)- \\
\mathrm{C} 9(122.03)\end{array}$ & $\begin{array}{l}\mathrm{H} 7(7.00)-\mathrm{H} 6(6.91) \\
\mathrm{H} 7(7.00)-\mathrm{H} 8(6.46)\end{array}$ & $\mathrm{H} 7(7.00)-\mathrm{H} 6(6.91)$ \\
\hline
\end{tabular}


$\mathrm{H} 7(7.00)-\mathrm{H} 8(6.46)$

\begin{tabular}{lccccc}
- & $\mathrm{C}-11$ & - & - & - \\
- & $\mathrm{C}-7$ & - & & - \\
$\mathrm{H}-14$ & $\mathrm{C}-18$ & $\mathrm{H} 14(7.43)-$ & $\mathrm{H} 14(7.43)-$ & - & - \\
- & $\mathrm{C}-16$ & $\mathrm{C} 18(160.60)$ & $\mathrm{C} 18(160.60)$ & & - \\
$\mathrm{H}-9$ & - & - & & - & - \\
$(\mathrm{N}-\mathrm{H})$ & & - & & \\
\hline 32 & 23 & & & &
\end{tabular}

Sup 4 Comparison between the chemical shift values of 1H NMR for mitragynine and silane-reduced-mitragynine

\begin{tabular}{|c|c|c|}
\hline \multirow[t]{2}{*}{ Parameters } & \multicolumn{2}{|c|}{ Chemical Shift, $\delta$ (ppm) } \\
\hline & Pure Mitragynine $^{15}$ & Silane-reduced-mitragynine \\
\hline$\overline{\mathrm{H}-18}$ & $0.87(3 \mathrm{H}, \mathrm{dd}, \mathrm{J}=7.5 \mathrm{~Hz})$ & $0.87\left(3 \mathrm{H}, \mathrm{dd}, \mathrm{J}=7.5 \mathrm{~Hz},-\mathrm{CH}_{3}\right)$ \\
\hline $\mathrm{H}-16$ & $1.64(1 \mathrm{H}, \mathrm{br}-\mathrm{d}, \mathrm{J}=11.3)$ & $1.19(1 \mathrm{H}, \mathrm{m},-\mathrm{CH})$ \\
\hline $\mathrm{H}-17 \alpha$ & $1.30\left(1 \mathrm{H}, \mathrm{m}, \mathrm{CH}_{2}\right)$ & $1.26\left(1 \mathrm{H}, \mathrm{m},-\mathrm{CH}_{2}\right)$ \\
\hline $\mathrm{H}-17 \beta$ & $1.75-1.80(2 \mathrm{H}, \mathrm{m})$ & $1.29\left(1 \mathrm{H}, \mathrm{m},-\mathrm{CH}_{2}\right)$ \\
\hline $\mathrm{H}-11 \alpha$ & $1.75-1.80(2 \mathrm{H}, \mathrm{m})$ & $1.67\left(1 \mathrm{H}, \mathrm{m},-\mathrm{CH}_{2}\right)$ \\
\hline $\mathrm{H}-11 \beta$ & $2.40-2.55(3 \mathrm{H}, \mathrm{m})$ & $1.77\left(1 \mathrm{H}, \mathrm{m},-\mathrm{CH}_{2}\right)$ \\
\hline $\mathrm{H}-4 \alpha$ & $2.90-3.00(2 \mathrm{H}, \mathrm{m})$ & $1.86\left(1 \mathrm{H}, \mathrm{m},-\mathrm{CH}_{2}\right)$ \\
\hline $\mathrm{H}-4 \beta$ & $3.04-3.07(3 \mathrm{H}, \mathrm{m})$ & $1.87\left(1 \mathrm{H}, \mathrm{m},-\mathrm{CH}_{2}\right)$ \\
\hline$H-19 \alpha$ & $2.40-2.55(3 \mathrm{H}, \mathrm{m})$ & $1.97\left(1 \mathrm{H}, \mathrm{m},-\mathrm{CH}_{2}\right)$ \\
\hline $\mathrm{H}-12$ & $3.04-3.07(3 \mathrm{H}, \mathrm{m})$ & $2.00(1 \mathrm{H}, \mathrm{m},-\mathrm{CH})$ \\
\hline $\mathrm{H}-19 \beta$ & $3.04-3.07(3 \mathrm{H}, \mathrm{m})$ & $2.04\left(1 \mathrm{H}, \mathrm{m},-\mathrm{CH}_{2}\right)$ \\
\hline $\mathrm{H}-3 \alpha$ & $2.40-2.55(3 \mathrm{H}, \mathrm{m})$ & $2.52\left(1 \mathrm{H}, \mathrm{m},-\mathrm{CH}_{2}\right)$ \\
\hline $\mathrm{H}-3 \beta$ & $2.90-3.00(2 \mathrm{H}, \mathrm{m})$ & $2.54\left(1 \mathrm{H}, \mathrm{m},-\mathrm{CH}_{2}\right)$ \\
\hline $\mathrm{H}-1$ & - & $3.02(1 \mathrm{H}, \mathrm{m},-\mathrm{CH})$ \\
\hline H-5 & - & $3.08(1 \mathrm{H}, \mathrm{m},-\mathrm{CH})$ \\
\hline $17-\mathrm{OCH}_{3}$ & $3.68\left(3 \mathrm{H}, \mathrm{s}, 22-\mathrm{OCH}_{3}\right)$ & $3.70\left(3 \mathrm{H}, \mathrm{s}, 17-\mathrm{OCH}_{3}\right)$ \\
\hline $19-\mathrm{OCH}_{3}$ & $3.78\left(3 \mathrm{H}, \mathrm{s}, 17-\mathrm{OCH}_{3}\right)$ & $3.73\left(3 \mathrm{H}, \mathrm{s}, 19-\mathrm{OCH}_{3}\right)$ \\
\hline 10-ОCH3 & $3.84\left(3 \mathrm{H}, \mathrm{s}, 9-\mathrm{OCH}_{3}\right)$ & $3.86\left(3 \mathrm{H}, \mathrm{s}, 10-\mathrm{OCH}_{3}\right)$ \\
\hline $\mathrm{H}-8$ & $6.41(1 \mathrm{H}, \mathrm{d}, \mathrm{J}=7.9)$ & $6.46(1 \mathrm{H}, \mathrm{d}, \mathrm{J}=7.7 \mathrm{~Hz}, \mathrm{Ar}-\mathrm{H})$ \\
\hline H-6 & $6.89(1 \mathrm{H}, \mathrm{d}, \mathrm{J}=7.9 \mathrm{~Hz})$ & $6.91(1 \mathrm{H}, \mathrm{d}, \mathrm{J}=8.1 \mathrm{~Hz}, \mathrm{Ar}-\mathrm{H})$ \\
\hline $\mathrm{H}-7$ & $6.91(1 \mathrm{H}, \mathrm{dd}, \mathrm{J}=7.9,7.9 \mathrm{~Hz})$ & $7.00(1 \mathrm{H}, \mathrm{t}, \mathrm{Ar}-\mathrm{H})$ \\
\hline $\mathrm{H}-14$ & $7.53(1 \mathrm{H}, \mathrm{s})$, & $7.43(1 \mathrm{H}, \mathrm{s}, \mathrm{Ar}-\mathrm{H})$ \\
\hline N-H (H9) & 7.68(1H,br-s,N-H) & $5.23(1 \mathrm{H}, \mathrm{br}-\mathrm{s}, \mathrm{N}-\mathrm{H})$ \\
\hline Solvent $\mathrm{CDCl} 3$ & 7.26 & 7.26 \\
\hline
\end{tabular}


Sup 5 Comparison between the chemical shift values of 13C NMR for Mitragynine and Silane-reduced-mitragynine

\begin{tabular}{lrr}
\hline Parameters & \multicolumn{2}{c}{ Chemical Shift, $\delta(\mathrm{ppm})$} \\
& Pure Mitragynine & \\
& & Silane-reduced-mitragynine \\
\hline C-1 & 133.69 & 66.82 \\
C-2 & 61.33 & 64.22 \\
C-3 & 53.85 & 53.55 \\
C-4 & 22.78 & 23.55 \\
C-5 & 107.91 & 34.32 \\
C-6 & 117.72 & 117.55 \\
C-7 & 154.49 & 154.53 \\
C-8 & 104.26 & 104.25 \\
C-9 & 121.91 & 122.03 \\
C-10 & 99.80 & 99.78 \\
C-11 & 137.33 & 137.34 \\
C-13 & 29.78 & 29.74 \\
C-14 & 31.99 & 39.80 \\
C-15 & 11.55 & 111.25 \\
C-18 & 160.65 & 160.60 \\
C-23 & 12.95 & 12.81 \\
C-22 & 14.22 & 19.25 \\
C-21 & 39.95 & 40.36 \\
C-20 & 57.77 & 57.33 \\
C-16 & 169.36 & 169.14 \\
C-17 & 51.45 & 51.36 \\
C-12 & 55.40 & 55.30 \\
C-19 & 61.65 & 57.33 \\
& & \\
\hline
\end{tabular}


Sup. 6 Medium pressure chromatography setting

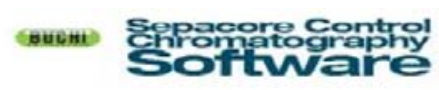

Method 10008.10 : Synthetic analogue of Mitragynine

\section{Separation}

Flowrate $[\mathrm{ml} / \mathrm{min}]$

Rack

Column

Pressure min.

$\max 50$

Tube Volume $[\mathrm{m}] \mathbf{6 0}$

Solvent Setup:

$$
\begin{array}{ll}
\text { Solvent B } & \text { Ethyl Acetate } \\
\text { Solvent A } & \text { Hexane }
\end{array}
$$

Conditioning

Flowrate $[\mathrm{ml} / \mathrm{min}]$

Rack

Column

20

FC30 (30 $500 \mathrm{ml})$

Glass Column 26/230-044036

$\checkmark$ Precolumn used

Pressure min 0 $\max 50$

Tube Volume [ml] $\mathbf{5 0}$

Solvent Setup:

Solvent B
Solvent A Hexane

\begin{tabular}{|c|c|c|c|}
\hline Start \&B & End $\% B$ & mii & $s$ \\
\hline 0 & o & 15 & 0 \\
\hline 2 & 2 & 15 & 0 \\
\hline 4 & 4 & 15 & 0 \\
\hline 6 & 6 & 25 & 0 \\
\hline 8 & 8 & 25 & o \\
\hline 10 & 10 & 10 & 0 \\
\hline 15 & 15 & 10 & 0 \\
\hline 25 & 25 & 5 & o \\
\hline 50 & 50 & 5 & 0 \\
\hline
\end{tabular}

\begin{tabular}{|c|c|c|c|}
\hline Start $7 B$ & End $\$ B$ & mil & $s$ \\
\hline 0 & 0 & 0 & 59 \\
\hline
\end{tabular}

Total Time: 00:00:59 
Sup. 7 Purification fractions and fractions containing pure silane reduced mitragynine

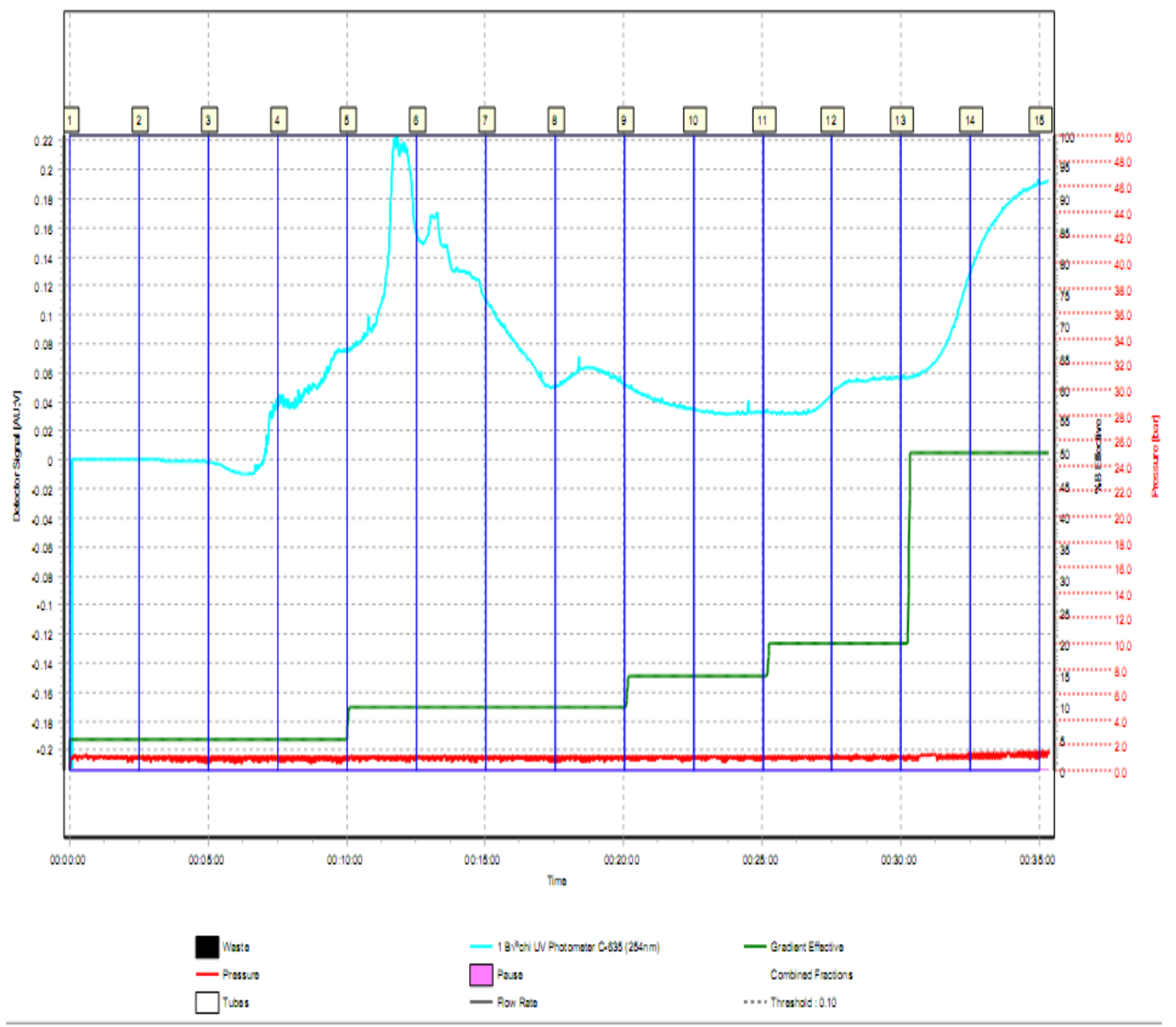


Sup. 8 Postulated mechanism of indole reduction
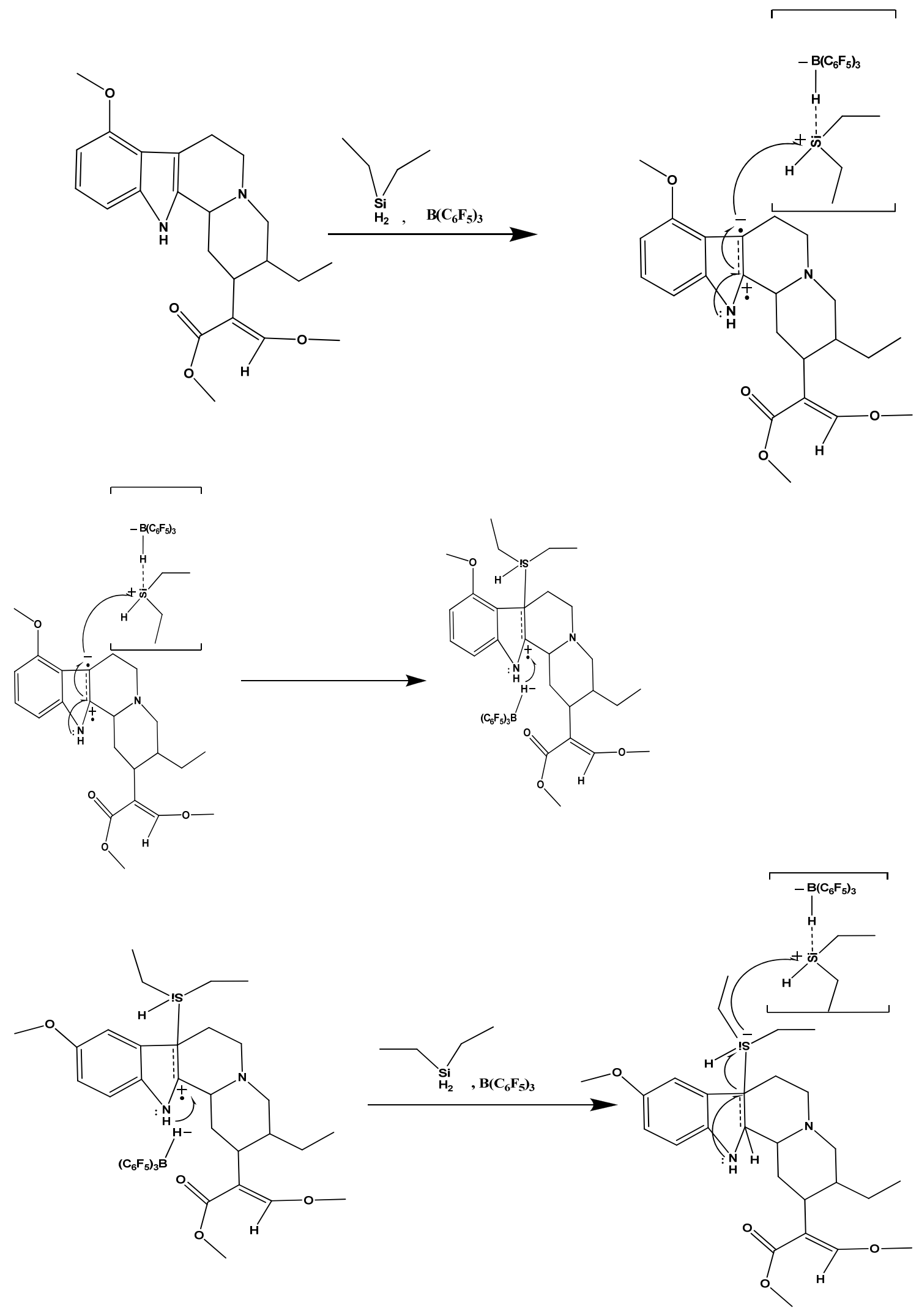


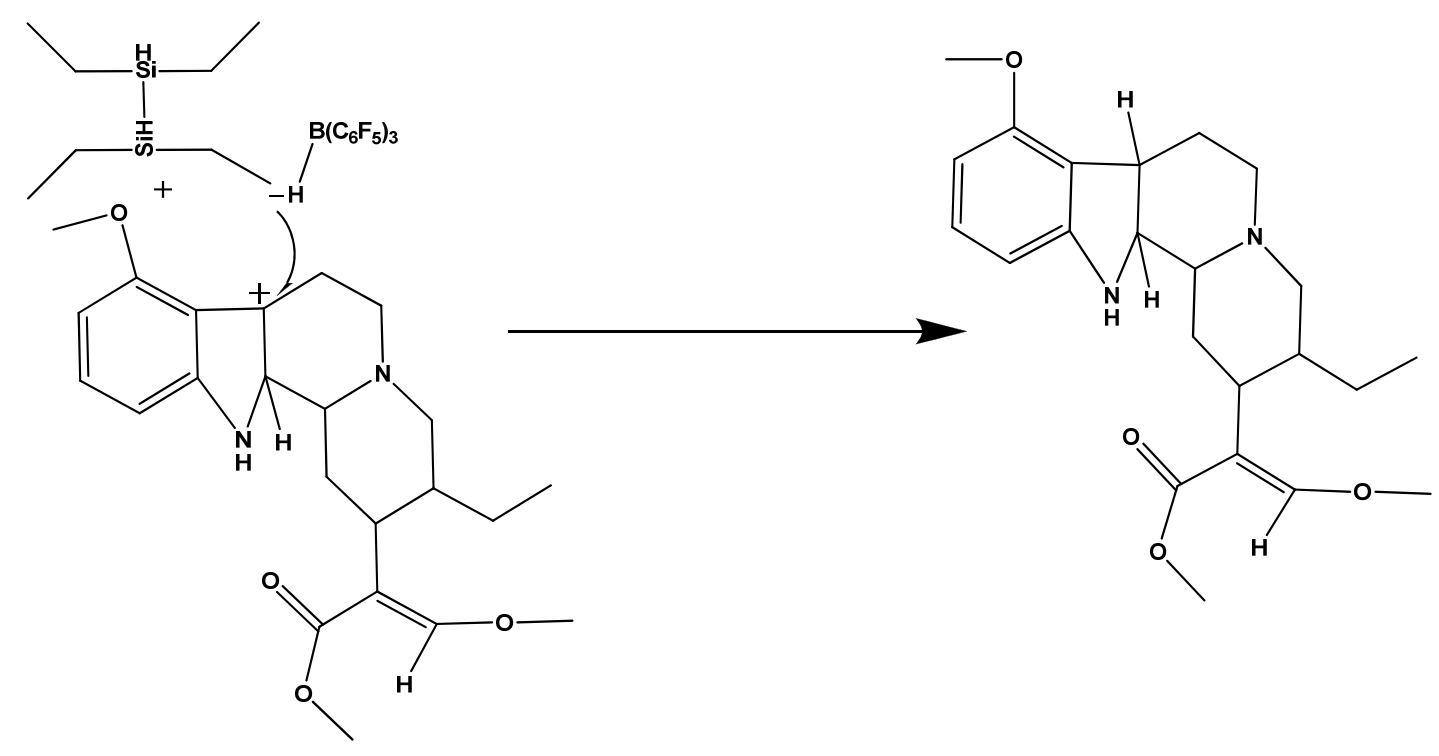

Sup. 9 DSC melting point of silane reduced mitragynine

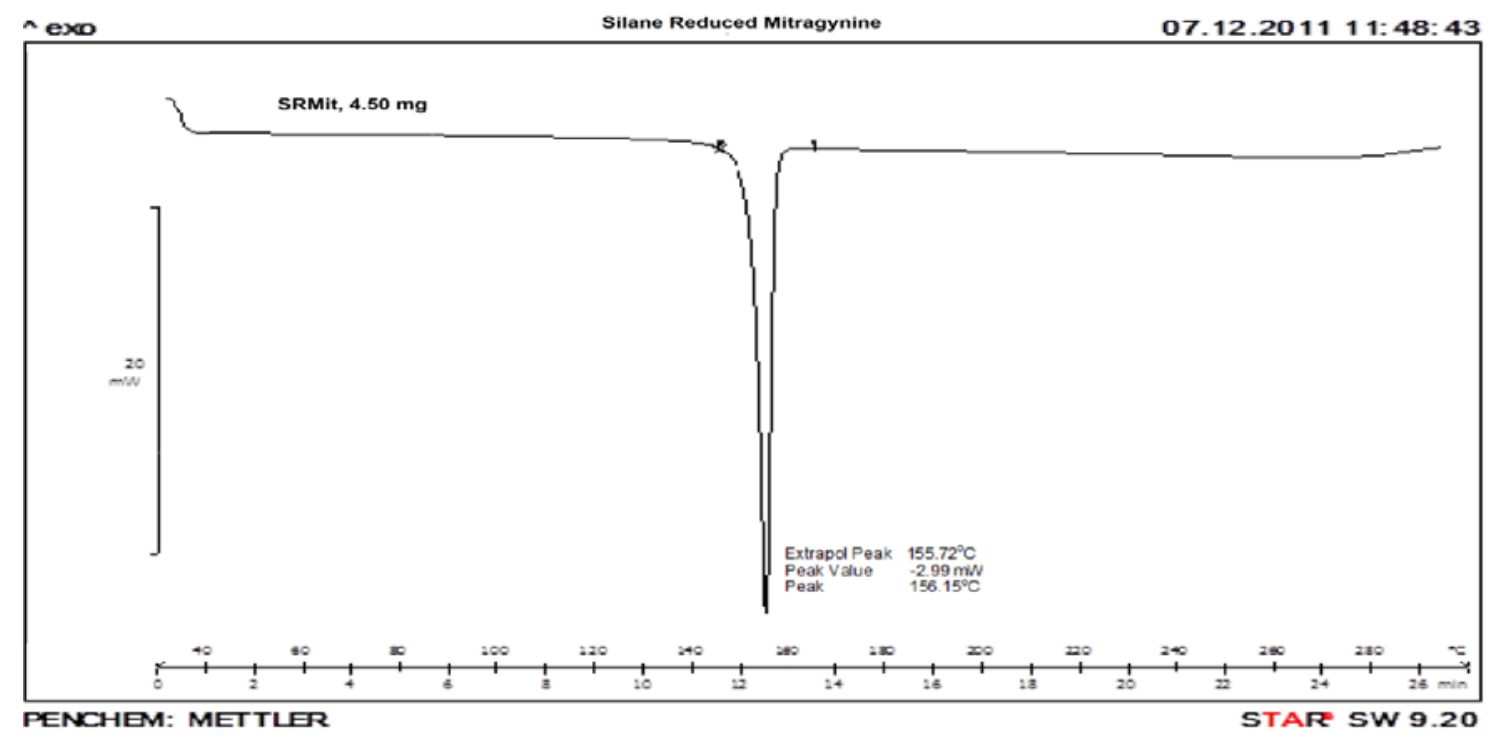


Sup. 10 Pure silane reduced mitragynine GC-MS peak

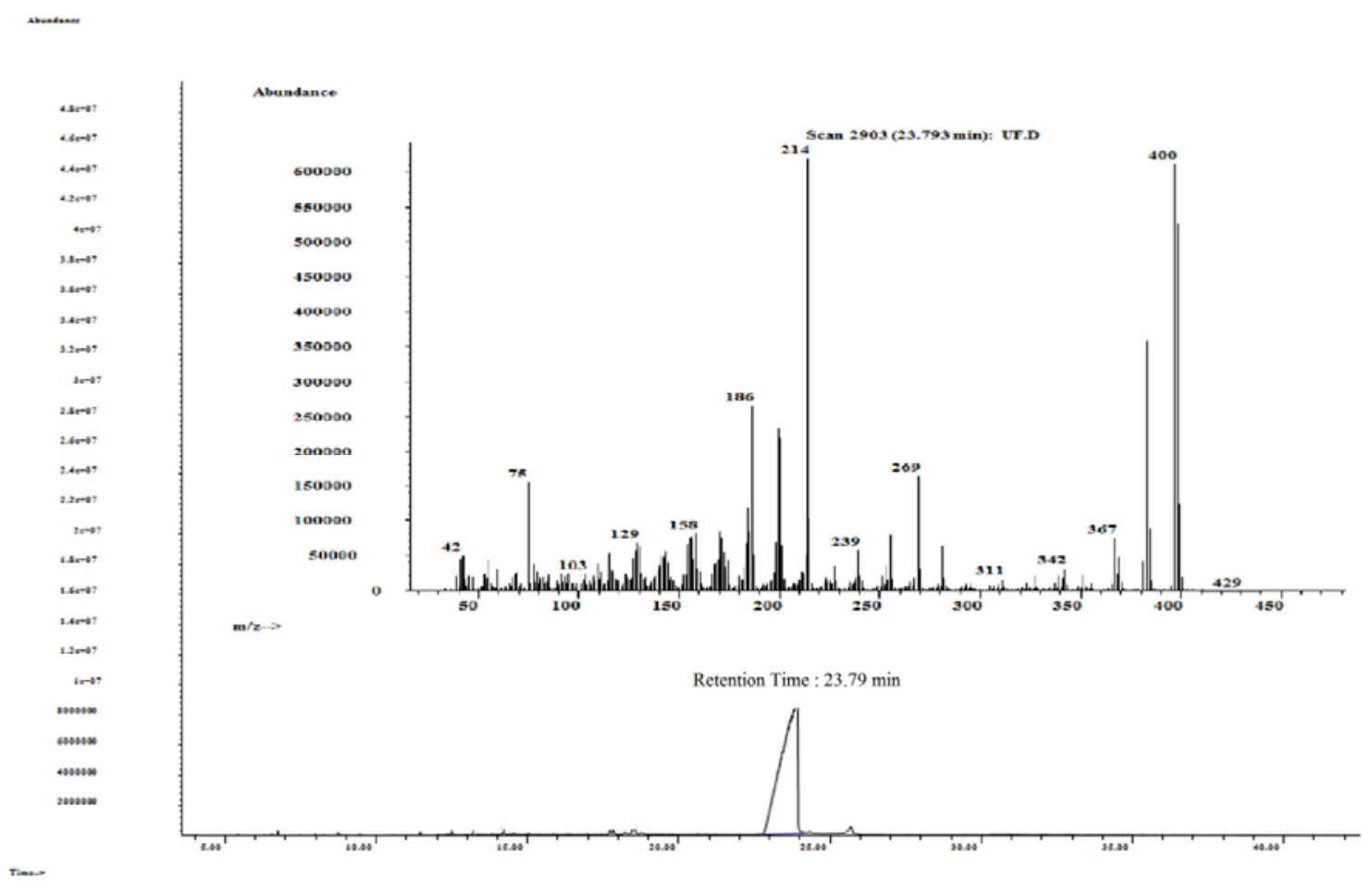


Sup. 11a 2D-HPTLC densitogramprofile of the crude reaction product with silane reduced mitragynine $(\mathrm{Rf}=0.52)$

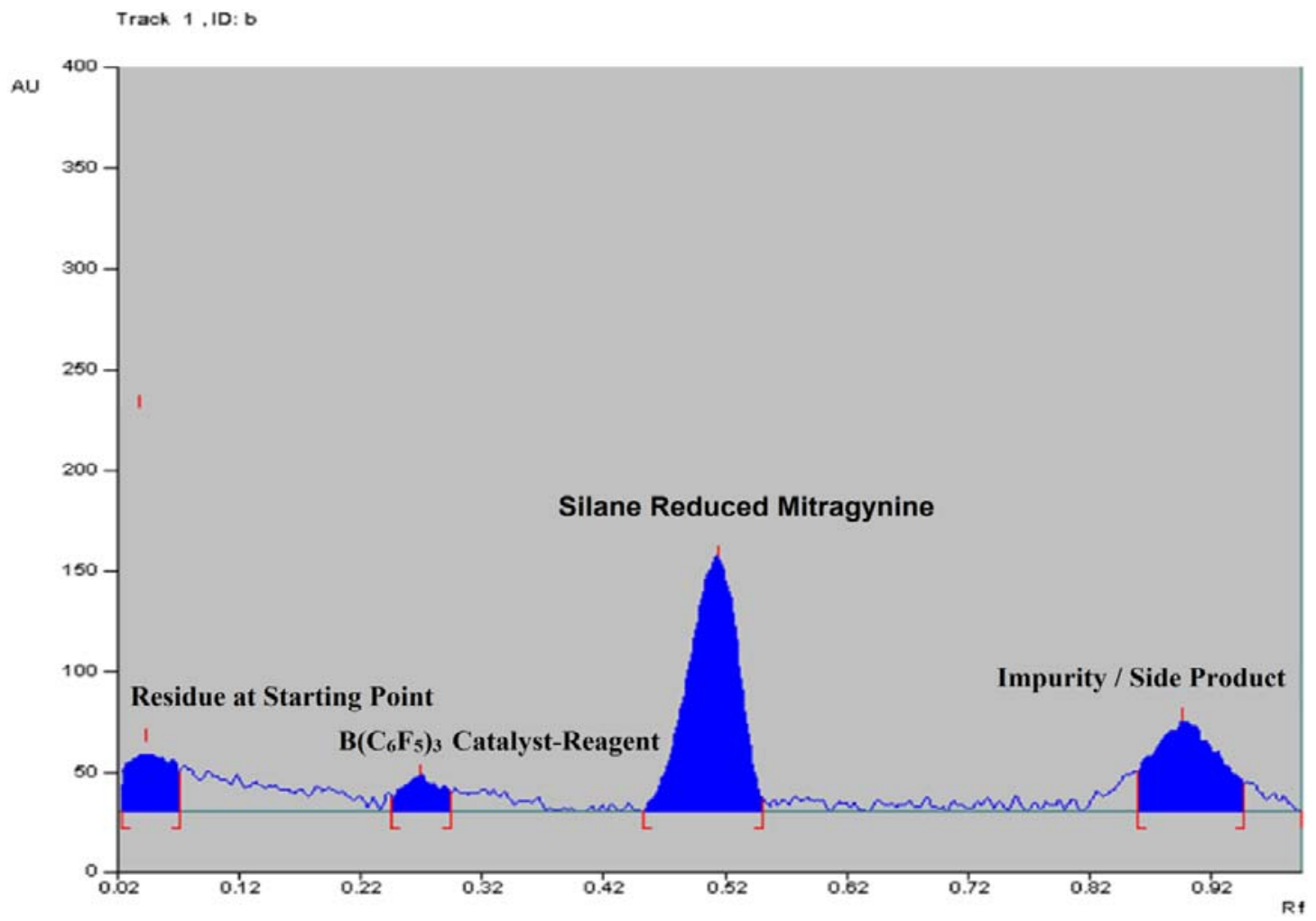

Sup. 11b 3D-HPTLC densitogram profile of the crude reaction product with silane reduced mitragynine $(\mathrm{Rf}=0.52)$

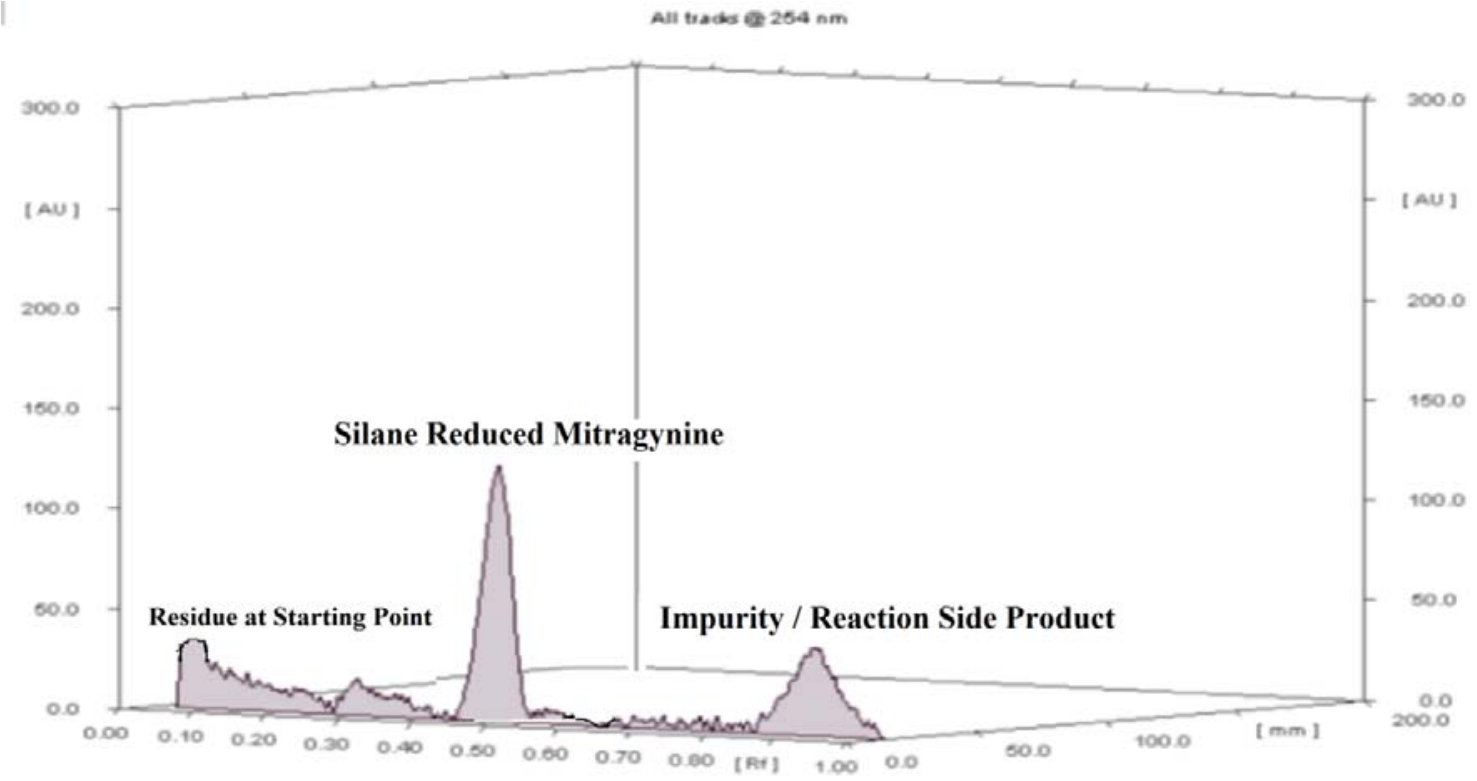


Sup. 12a\&12b Silane Reduced Mitragynine (Proton Numbering System and Carbon Numbering System)

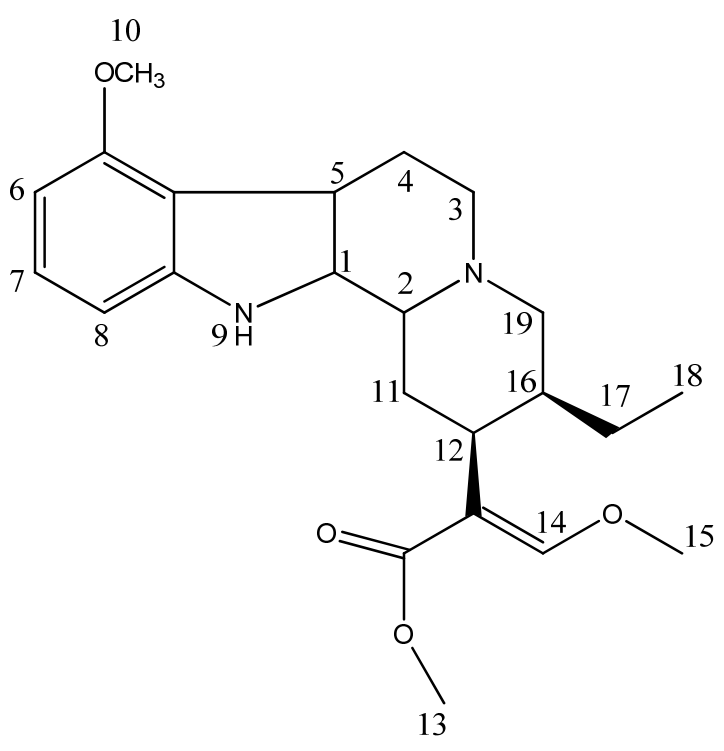

Fig. 8a : Silane Reduced Mitragynine (Proton Numbering System)

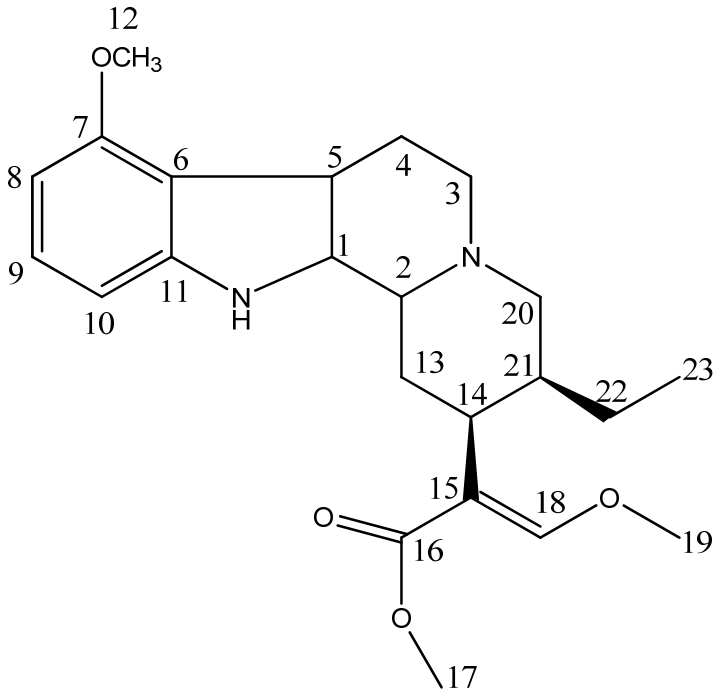

Fig. $8 \mathrm{~b}$ : Silane Reduced Mitragynine (Carbon Numbering System) 